\title{
Characterizing K2 Planet Discoveries: A Super- Earth Transiting the Bright K Dwarf Hip 116454
}

\section{Citation}

Vanderburg, Andrew, Benjamin T. Montet, John Asher Johnson, Lars A. Buchhave, Li Zeng, Francesco Pepe, Andrew Collier Cameron, et al. 2015. "Characterizing K2 Planet Discoveries: A Super-Earth Transiting the Bright K Dwarf Hip 116454." The Astrophysical Journal 800 (1) (February 9): 59. doi:10.1088/0004-637x/800/1/59.

\section{Published Version}

doi:10.1088/0004-637X/800/1/59

\section{Permanent link}

http://nrs.harvard.edu/urn-3:HUL.InstRepos:30781104

\section{Terms of Use}

This article was downloaded from Harvard University's DASH repository, and is made available under the terms and conditions applicable to Other Posted Material, as set forth at http:// nrs.harvard.edu/urn-3:HUL.InstRepos:dash.current.terms-of-use\#LAA

\section{Share Your Story}

The Harvard community has made this article openly available.

Please share how this access benefits you. Submit a story.

\section{Accessibility}




\title{
CHARACTERIZING K2 PLANET DISCOVERIES: A SUPER-EARTH TRANSITING THE BRIGHT K DWARF HIP 116454
}

\author{
Andrew Vanderburg ${ }^{1,28}$, Benjamin T. Montet ${ }^{1,2,28}$, John Asher Johnson $^{1,29}$, Lars A. BuchHave ${ }^{1}$, Li Zeng $^{1}$, \\ Francesco Pepe ${ }^{3}$, Andrew Collier Cameron ${ }^{4}$, David W. Latham ${ }^{1}$, Emilio Molinari ${ }^{5,6}$, StéPhane Udry $^{3}$, \\ Christophe Lovis $^{3}$, Jaymie M. Matthews $^{7}$, Chris Cameron $^{8}$, Nicholas Law $^{9}$, Brendan P. Bowler ${ }^{2,30}$, Ruth Angus ${ }^{1,10}$, \\ Christoph Baranec ${ }^{11}$, Allyson Bieryla ${ }^{1}$, Walter Boschin ${ }^{5}$, David Charbonneau ${ }^{1}$, Rosario Cosentino ${ }^{5}$, \\ Xavier Dumusque ${ }^{1}$, Pedro Figueira ${ }^{12,13}$, David B. Guenther ${ }^{14}$, Avet Harutyunyan ${ }^{5}$, Coel Hellier $^{15}$, \\ Rainer Kuschnig $^{16}$, Mercedes Lopez-Morales ${ }^{1}$, Michel Mayor ${ }^{3}$, Giusi Micela ${ }^{17}$, Anthony F. J. Moffat ${ }^{18,19}$, \\ Marco Pedani $^{5}$, David F. Phillips ${ }^{1}$, Giampaolo Piotto ${ }^{5,6}$, Don Pollacco $^{20}$, Didier Queloz ${ }^{21}$, Ken Rice $^{22}$, Reed Riddle $^{2}$, \\ Jason F. Rowe ${ }^{23,24}$, Slavek M. Rucinski ${ }^{25}$, Dimitar Sasselov ${ }^{1}$, Damien Ségransan ${ }^{3}$, Alessandro Sozzetti ${ }^{26}$, \\ AndreW Szentgyorgyi ${ }^{1}$, Chris WATSON ${ }^{27}$, AND WERnER W. Weiss ${ }^{16}$ \\ ${ }^{1}$ Harvard-Smithsonian Center for Astrophysics, Cambridge, MA 02138, USA; avanderburg@cfa.harvard.edu \\ ${ }^{2}$ California Institute of Technology, Pasadena, CA 91125, USA \\ ${ }^{3}$ Observatoire Astronomique de l'Université de Genève, 51 chemin des Maillettes, CH-1290 Versoix, Switzerland \\ ${ }^{4}$ SUPA, School of Physics and Astronomy, University of St Andrews, North Haugh, St Andrews, Fife KY16 9SS, UK \\ ${ }^{5}$ INAF-Fundación Galileo Galilei, Rambla José Ana Fernández Pérez, 7, E-38712 Breña Baja, Spain \\ ${ }^{6}$ INAF-IASF Milano, via Bassini 15, I-20133 Milano, Italy \\ ${ }^{7}$ University of British Columbia, Vancouver, BC V6T1Z1, Canada \\ ${ }^{8}$ Cape Breton University, 1250 Grand Lake Road, Sydney NS B1P 6L2, Canada \\ ${ }^{9}$ University of North Carolina at Chapel Hill, Chapel Hill, NC 27599, USA \\ ${ }^{10}$ University of Oxford, Oxford, UK \\ ${ }^{11}$ University of Hawai 'i at Mānoa, Hilo, HI 96720, USA \\ ${ }^{12}$ Centro de Astrofísica, Universidade do Porto, Rua das Estrelas, 4150-762 Porto, Portugal \\ ${ }^{13}$ Instituto de Astrofísica e Ciências do Espaço, Universidade do Porto, CAUP, Rua das Estrelas, PT4150-762 Porto, Portugal \\ ${ }^{14}$ St. Mary's University, Halifax, NS B3H 3C3, Canada \\ ${ }^{15}$ Astrophysics Group, Keele University, Staffordshire ST5 5BG, UK \\ ${ }^{16}$ Institut für Astronomie, Universität Wien, Türkenschanzstrasse 17, A-1180 Wien, Austria \\ ${ }^{17}$ INAF-Osservatorio Astronomico di Palermo, Piazza del Parlamento 1, I-90124 Palermo, Italy \\ ${ }^{18}$ Univ de Montréal, C.P. 6128, Succ. Centre-Ville, Montréal, QC H3C 3J7, Canada \\ ${ }^{19}$ Obs. du mont Mégantic, Notre-Dame-des-Bois, QC J0B 2E0, Canada \\ ${ }^{20}$ Department of Physics, University of Warwick, Gibbet Hill Road, Coventry CV4 7AL, UK \\ ${ }^{21}$ Cavendish Laboratory, J J Thomson Avenue, Cambridge CB3 0HE, UK \\ ${ }^{22}$ SUPA, Institute for Astronomy, Royal Observatory, University of Edinburgh, Blackford Hill, Edinburgh EH93HJ, UK \\ ${ }^{23}$ SETI Institute, 189 Bernardo Avenue, Mountain View, CA 94043, USA \\ ${ }^{24}$ NASA Ames Research Center, Moffett Field, CA 94035, USA \\ ${ }^{25}$ University of Toronto, 50 St. George Street, Toronto, ON M5S 3H4, Canada \\ ${ }^{26}$ INAF-Osservatorio Astrofisico di Torino, Via Osservatorio 20, I-10025 Pino Torinese, Italy \\ ${ }^{27}$ Astrophysics Research Centre, School of Mathematics and Physics, Queens University Belfast, Belfast BT7 1NN, UK \\ Received 2014 October 20; accepted 2014 December 12; published 2015 February 9
}

\begin{abstract}
We report the first planet discovery from the two-wheeled Kepler (K2) mission: HIP 116454 b. The host star HIP 116454 is a bright $(V=10.1, K=8.0) \mathrm{K} 1$ dwarf with high proper motion and a parallax-based distance of $55.2 \pm 5.4 \mathrm{pc}$. Based on high-resolution optical spectroscopy, we find that the host star is metal-poor with $[\mathrm{Fe} / \mathrm{H}]=-0.16 \pm 0.08$ and has a radius $R_{\star}=0.716 \pm 0.024 R_{\odot}$ and mass $M_{\star}=0.775 \pm 0.027 M_{\odot}$. The star was observed by the Kepler spacecraft during its Two-Wheeled Concept Engineering Test in 2014 February. During the 9 days of observations, K2 observed a single transit event. Using a new K2 photometric analysis technique, we are able to correct small telescope drifts and recover the observed transit at high confidence, corresponding to a planetary radius of $R_{p}=2.53 \pm 0.18 R_{\oplus}$. Radial velocity observations with the HARPS-N spectrograph reveal a $11.82 \pm 1.33 M_{\oplus}$ planet in a 9.1 day orbit, consistent with the transit depth, duration, and ephemeris. Follow-up photometric measurements from the MOST satellite confirm the transit observed in the K2 photometry and provide a refined ephemeris, making HIP $116454 \mathrm{~b}$ amenable for future follow-up observations of this latest addition to the growing population of transiting super-Earths around nearby, bright stars.
\end{abstract}

Key words: planets and satellites: detection - techniques: photometric

Supporting material: FITS file

\section{INTRODUCTION}

After four years of nearly continuous photometric monitoring and thousands of planet discoveries (e.g., Borucki et al. 2011;

\footnotetext{
${ }^{28}$ NSF Graduate Research Fellow.

${ }^{29}$ David, and Lucile Packard Fellow.

${ }^{30}$ Caltech Joint Center for Planetary Astronomy Fellow.
}

Howard et al. 2012; Muirhead et al. 2012; Batalha et al. 2013; Barclay et al. 2013; Morton \& Swift 2014), the primary Kepler mission came to an end in 2013 May with the failure of the second of four reaction wheels used to stabilize the spacecraft. Without at least three functioning reaction wheels, the spacecraft is unable to achieve the fine pointing necessary for high photometric precision on the original target field. However, 
an extended mission called $\mathrm{K} 2$ was enabled by pointing along the ecliptic plane and balancing the spacecraft against solar radiation pressure to mitigate the instability caused by the failed reaction wheels. The recently extended K2 mission enables renewed opportunities for transit science on a new set of bright target stars, albeit with somewhat reduced photometric precision compared to the original Kepler mission (Howell et al. 2014).

Searching for transiting exoplanets around bright, nearby stars is important because measuring the precise masses and radii of transiting planets allows for characterization of their interior structures and atmospheres (Charbonneau et al. 2002; Rogers 2014; Knutson et al. 2014; Teske et al. 2013; Kreidberg et al. 2014). This is particularly desirable for planets with masses intermediate to those of the Earth and Uranus, commonly referred to as super-Earths, because no such planet exists in our solar system (Valencia et al. 2006). However, while the radii of Kepler planets are often measured to high precision (Ballard et al. 2014), their masses are generally unknown because the host stars are faint $(V>12)$ and exposure times needed for radial velocity (RV) measurements are prohibitive for all but the brightest Kepler planet candidates (e.g., Dumusque et al. 2014; Marcy et al. 2014).

Preparations for the extended two-wheeled Kepler mission included a 9 day test of the new observing mode in February of 2014. After the data were released to the public, Vanderburg \& Johnson (2014, hereafter VJ14) presented a photometric reduction technique that accounts for the motion of the spacecraft, improves photometric precision of raw $\mathrm{K} 2$ data by a factor of $2-5$, and enables photometric precision comparable to that of the original Kepler mission.

While the data collected during the engineering test were intended primarily as a test of the new spacecraft operating mode, an inspection of light curves produced with this technique nonetheless revealed a single transit event in engineering data taken of HIP 116454. In this paper, we provide an analysis of that light curve along with archival and follow-up spectroscopy, archival and adaptive optics imaging, RV measurements from the HARPS-N spectrograph, and photometric observations from the Wide Angle Search for Planets (WASP) survey and the Microvariability and Oscillations of STars (MOST) space telescope. These measurements allow us to verify and characterize the first planet discovered by the two-wheeled Kepler mission, a new transiting super-Earth orbiting the bright, nearby, highproper-motion K dwarf HIP 116454.

\section{DATA AND ANALYSIS}

\subsection{K2 Photometry}

HIP 116454 and nearly 2,000 other stars were observed by the Kepler spacecraft from 2014 February 4 until 2014 February 12 during the Kepler Two-Wheel Concept Engineering Test. After the first 2.5 days of the test, Kepler underwent a large, intentional adjustment to its pointing to move its target stars to the center of their apertures, where they stayed for the last 6.5 days of the test. We downloaded the full engineering test data set from the Mikulski Archive for Space Telescopes (MAST) and reduced the Kepler target pixel files as described in VJ14.

In brief, we extracted raw aperture photometry and image centroid positions from the Kepler target pixel files. Raw K2 photometry is dominated by jagged events corresponding to the motion of the spacecraft, as Kepler's pointing drifts because of solar radiation pressure and is periodically corrected with thrusters. For the last 6.5 days after the large pointing tweak, we
Table 1

Astrometric and Photometric Properties of HIP 116454

\begin{tabular}{|c|c|c|c|}
\hline Parameter & Value & Uncertainty & Source \\
\hline$\alpha(\mathrm{J} 2000)$ & 233549.28 & $\ldots$ & Hipparcos $^{\mathrm{a}}$ \\
\hline$\delta(\mathrm{J} 2000)$ & +002643.86 & $\ldots$ & Hipparcos \\
\hline 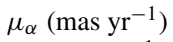 & -238.0 & 1.7 & Hipparcos \\
\hline$\mu_{\delta}\left(\operatorname{mas}_{\mathrm{yr}} \mathrm{r}^{-1}\right)$ & -185.9 & 0.9 & Hipparcos \\
\hline$\pi$ (mas) & 18.1 & 1.72 & Hipparcos \\
\hline B & 11.08 & 0.01 & Tycho \\
\hline$V$ & 10.190 & 0.009 & Tycho ${ }^{\mathrm{b}}$ \\
\hline$R$ & 9.71 & 0.03 & TASS $^{\mathrm{c}}$ \\
\hline$I$ & 9.25 & 0.03 & TASS \\
\hline$u$ & 14.786 & 0.02 & $\operatorname{SDSS}^{\mathrm{d}}$ \\
\hline$g$ & 10.837 & 0.02 & SDSS \\
\hline$r$ & 9.908 & 0.02 & SDSS \\
\hline$i$ & 9.680 & 0.02 & SDSS \\
\hline$J$ & 8.60 & 0.02 & $2 \mathrm{MASS}^{\mathrm{e}}$ \\
\hline$H$ & 8.14 & 0.03 & 2MASS \\
\hline$K_{S}$ & 8.03 & 0.02 & 2MASS \\
\hline
\end{tabular}

Notes.

a van Leeuwen (2007).

b Egret et al. (1994).

c Richmond et al. (2000).

d Abazajian et al. (2009).

e Skrutskie et al. (2006).

removed the systematics due to the motion of the spacecraft by correlating the measured flux with the image centroid positions measured from photometry. We essentially produced a "self flat field" (SFF) similar to those produced by, for instance, Ballard et al. (2010), for analysis of Spitzer photometry. We fit a piecewise linear function to the measured dependence of flux on centroid position, with outlier exclusion to preserve transit events, and removed the dependence on centroid position from the raw light curve. Similar to VJ14, we excluded data points taken while Kepler's thrusters were firing from our reduced light curves because these data were typically outliers from the corrected light curves. For HIP 116454, the median absolute deviation (MAD) of the 30-minute-long cadence data points improved from $\simeq 500$ parts per million (ppm) for the raw light curve to $\simeq 50 \mathrm{ppm}$ for the SFF light curve.

Visual inspection of light curves from the $\simeq 2000$ targets observed during the engineering test revealed a 1 millimagnitude (mmag) deep candidate transit in photometry of HIP 116454, designated EPIC 60021410 by the Kepler team. HIP 116454's photometric and astrometric measurements are summarized in Table 1. Raw and corrected K2 photometry for HIP 116454 are shown in Figure 1. We fit a Mandel \& Agol (2002) model to the transit and measured a total duration of approximately $2.25 \mathrm{hr}$ and a planet-to-star radius ratio of approximately 0.03 . Unfortunately, the data point during transit ingress happened during a thruster firing event and was excluded by our pipeline. This particular data point does not appear to be anomalous, but we choose to exclude it to minimize risk of contaminating the transit with an outlier. Slow photometric variability, presumably due to starspot modulation, is evident in the K2 light curve at the subpercent level.

We also performed a similar SFF correction to the data taken in the 2.5 days of data before the large pointing tweak. Even though the resulting data quality is somewhat worse, we are able to confidently exclude any other events of a similar depth during that time.

Because K2 only observed one transit event, we were not able to measure a precise orbital period for the planet candidate. 


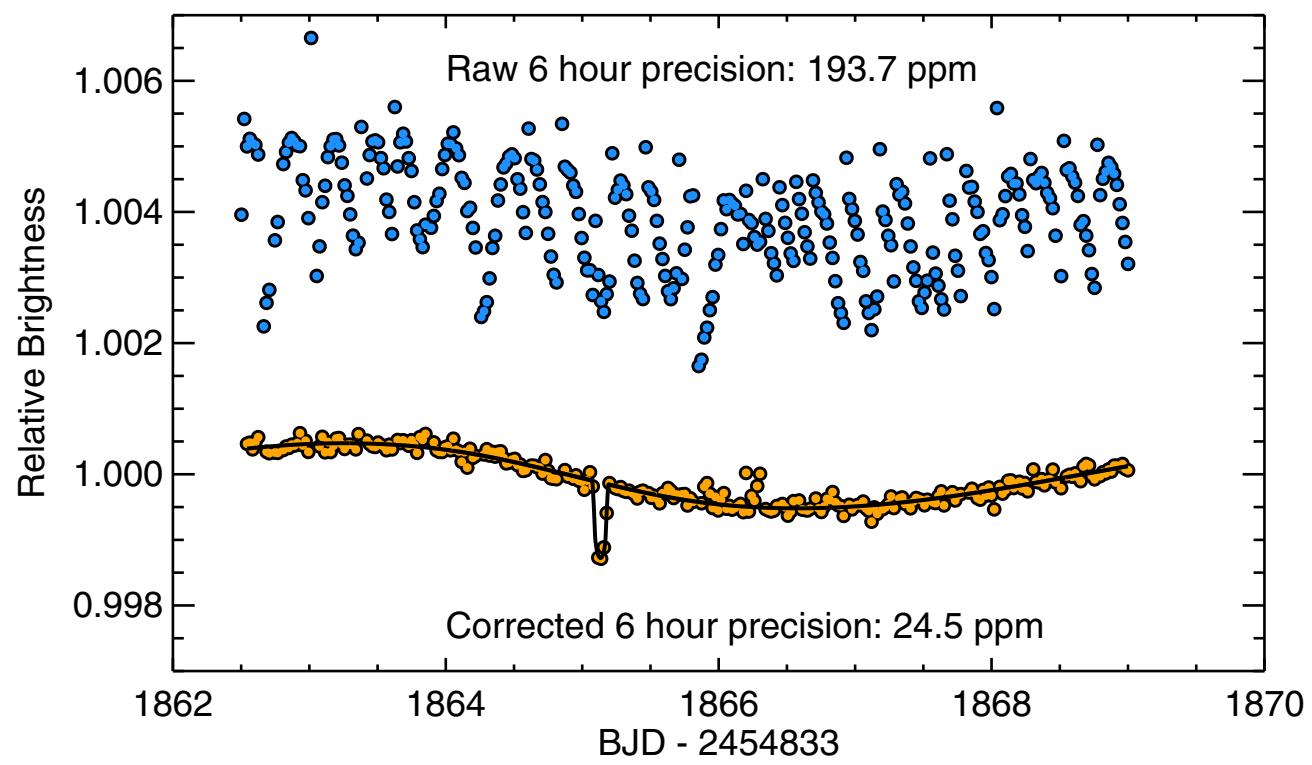

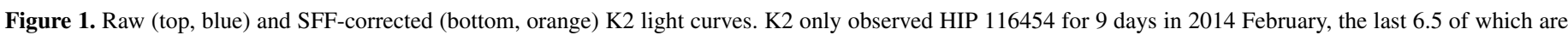

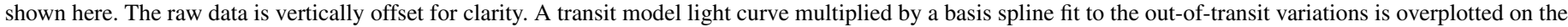
corrected K2 data. The $6 \mathrm{hr}$ photometric precision on this target (as defined by VJ14) improves by a factor of seven as a result of the SFF processing.

Nonetheless, we were able to put rough constraints on the orbital period from our knowledge of the transit duration and estimates of the stellar properties. The 9 day time baseline of the K2 observations allowed us to constrain the period of the candidate transiting planet to be greater than 5 days. To put a rough upper bound on the allowed planet period, we compared the transit duration of the candidate transit around HIP 116454 to the distribution of transit durations from the ensemble of Kepler planet candidates (retrieved from the NASA Exoplanet Archive; Akeson et al. 2013). We found that of the 413 Kepler planet candidates with transit durations between 2 and $2.5 \mathrm{hr}$, $93 \%$ had orbital periods shorter than 20 days. Because transit duration is a function of the mean stellar density, we repeated this calculation while restricting the sample to the 64 planet candidates with transit durations between 2 and $2.5 \mathrm{hr}$ and hoststar effective temperatures within $200 \mathrm{~K}$ of HIP 116454 . We find similarly that $94 \%$ of these candidates had orbital periods shorter than 20 days.

\subsection{Imaging}

\subsubsection{Archival Imaging}

We used a combination of modern and archival imaging to limit potential false-positive scenarios for the transit event. HIP 116454 was observed in the National Geographic Society-Palomar Observatory Sky Survey (POSS-I) on 1951 November 28. HIP 116454 has a proper motion of 303 mas yr $^{-1}$ (van Leeuwen 2007) and therefore has moved nearly 20 arcsec with respect to background sources since being imaged in POSS-I. Inspection of the POSS-I image reveals no background objects within the K2 aperture used in our photometric reduction. We show the POSS-I blue image overlaid with the K2 photometric aperture in Figure 2(a). The POSS-I survey has a limiting magnitude of 21.1 in the blue bandpass (Abell 1955), 10 mag fainter than HIP 116454. The depth of the detected transit is $0.1 \%$, so if a background eclipsing binary were responsible, the depth would correspond to a total eclipse of a star 7.5 mag fainter. A low-proper-motion background star such as that would have readily been detected in POSS-I imaging. We conclude that our aperture is free of background objects whose eclipses could masquerade as planet transits.

The POSS-I imaging also reveals a companion star about 8 arcsec to the southwest of HIP 116454. The companion is not fully resolved in POSS-I because the photographic plate was saturated by the bright primary star, but an asymmetry in the stellar image is visible. HIP 116454 was also observed during the Second Palomar Observatory Sky Survey (POSS-II) on 1992 August 31. Improvements in photographic plate technology over the previous $40 \mathrm{yr}$ allowed the companion star to be resolved. The companion shares a common proper motion with the primary at a projected distance of $\simeq 500 \mathrm{AU}$, so we conclude that the two stars are a gravitationally bound visual binary system.

\subsubsection{Modern Imaging}

HIP 116454 was observed during the Sloan Digital Sky Survey (SDSS), and the secondary star was detected (Abazajian et al. 2009). The secondary star falls on a diffraction spike caused by the much brighter primary star, but the SDSS pipeline flagged its photometry as "acceptable." The SDSS photometry indicates that the secondary star is 6-7 mag fainter than the primary, depending on the filter, so because the two stars are gravitationally associated, the secondary must be intrinsically much fainter than the K-dwarf primary. This implies that the companion must either be a late $\mathrm{M}$ dwarf or a white dwarf. The SDSS colors are relatively flat, indicating a hot star. To quantify this, we fit the ugri SDSS colors to a blackbody model, excluding $z$ because of its low throughput and assuming photometric errors of $5 \%$. We included no corrections for extinction because of the proximity of the target and our ability to accurately predict broadband photometry using stellar models in Section 3.1.2. We find that the data are best described by an object radiating at a temperature of $T_{\mathrm{WD}}=7500 \pm 200 \mathrm{~K}$. We used the Stefan-Boltzmann law combined with the Hipparcos parallax and derived the temperature to estimate a radius of $R_{\mathrm{WD}}=1.2 \pm 0.1 R_{\oplus}$, which is consistent with our white dwarf hypothesis. Using a simple analytic white dwarf cooling law (Mestel 1952; Gänsicke 1997), we estimate a cooling age of 
(a) POSS-I Blue: 1951

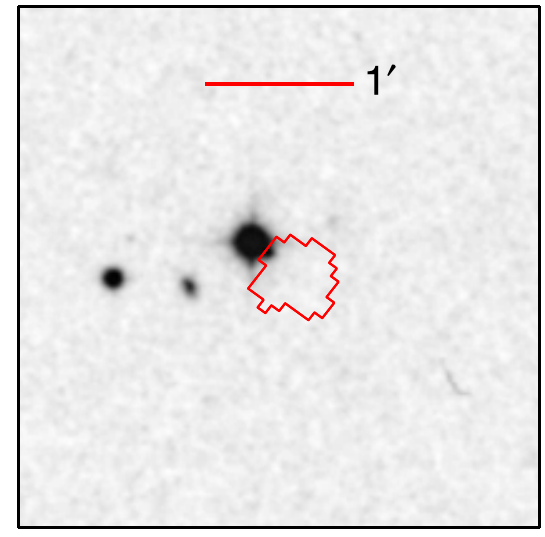

(d) POSS-I Blue: 1951, Zoomed

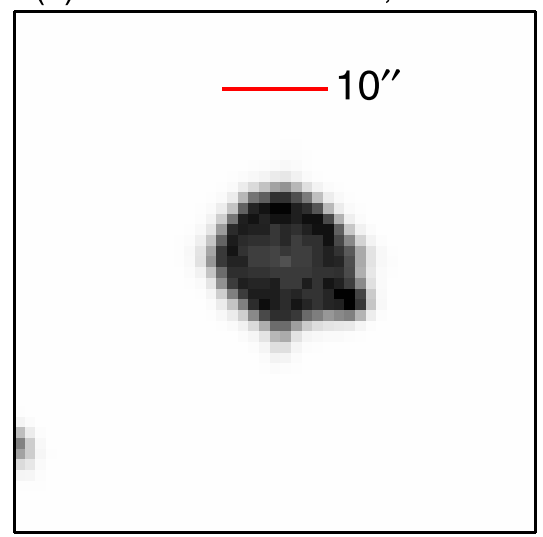

(b) POSS-II Blue: 1992

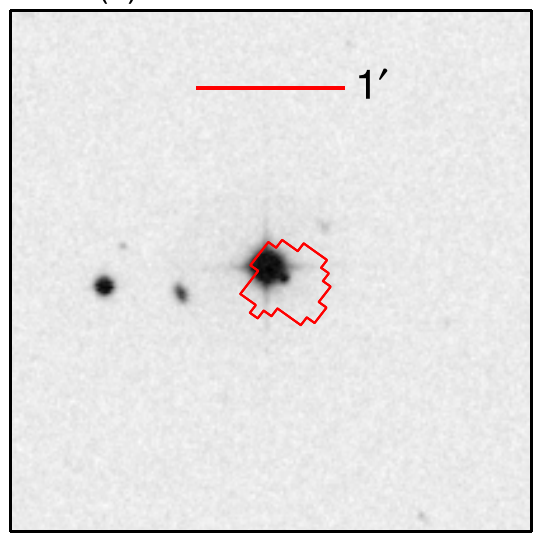

(e) NIRC2 Ks-band: 2014

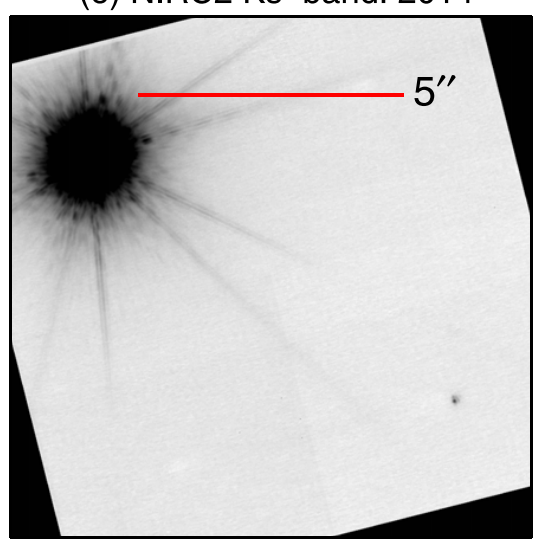

(c) K2: 2014

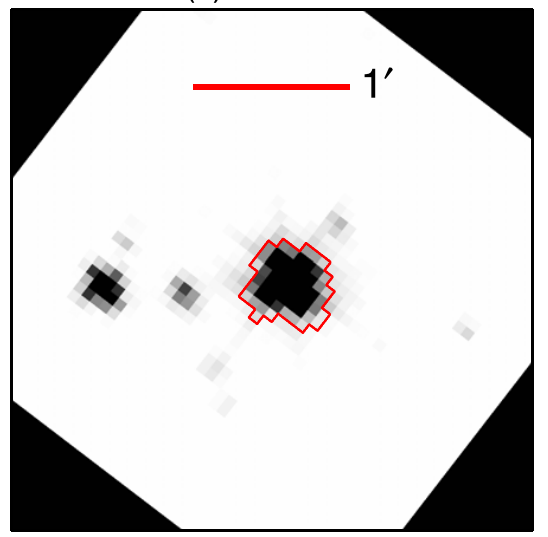

(f) Robo-AO LP600: 2014

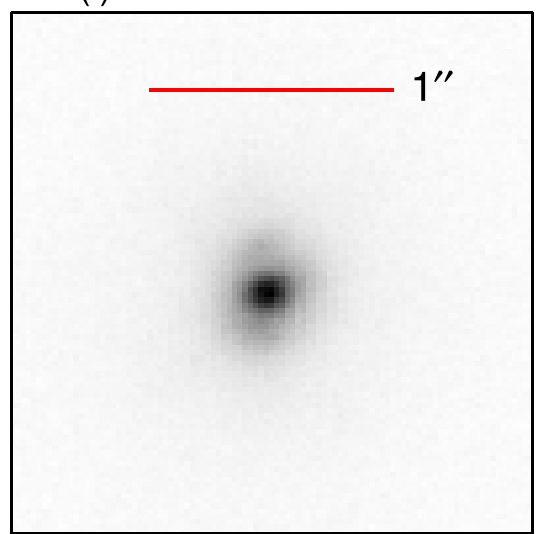

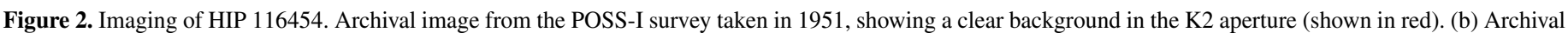

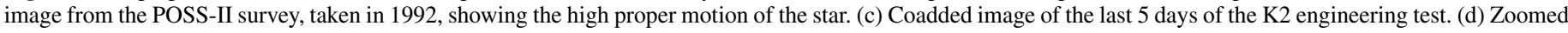

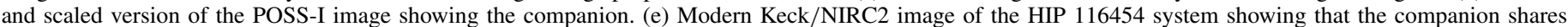

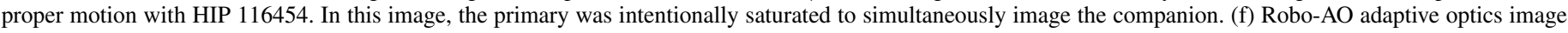

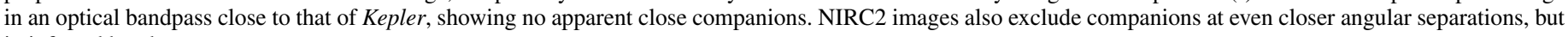
in infrared bandpasses.

the white dwarf of $t_{\text {cool }} \sim 1.3$ Gyr. The formal uncertainty on the cooling age is $0.2 \mathrm{Gyr}$, but this neglects uncertainties that are due to the unknown composition of the white dwarf and inaccuracies in the simple model. The true uncertainty on this quantity is likely on the order of a factor of two (van Horn 1971). The cooling age of the white dwarf is a lower limit on the age of the system, and the total age of the system is the sum of the main sequence lifetime of the progenitor and the white dwarf's cooling age.

The secondary star is close enough to the primary that it is blended in the K2 image and is bright enough that if it were a totally eclipsing binary, it could cause the transit event we observed. This situation is unlikely because the duration and minimum period of the event are generally inconsistent with an object eclipsing a white dwarf. With the baseline of K2 data, we can exclude orbital periods shorter than 5 days. While 5-dayperiod companions eclipsing main-sequence stars are common and have relatively high transit probabilities, the probability of a transit or eclipse goes as $P \propto R_{\star}$ at a given stellar mass and orbital period. Furthermore, in order for an Earth-sized object eclipsing a white dwarf to have an eclipse duration of $2 \mathrm{hr}$, the orbital period would have to be roughly $600 \mathrm{yr}$ in the case of a circular orbit and impact parameter $b=0$. Even with a highly elliptical orbit transiting at apastron, which is a priori unlikely, the orbital period would be on the order of centuries, and the semimajor axis would be roughly $50 \mathrm{AU}$. The probability of an orbit such as that eclipsing the white dwarf is $P \sim\left(R_{\star}+R_{p}\right) / a \sim 10^{-6}$, where $a$ is the semimajor axis and $R_{p}$ is the radius of the occulting body. In the worst-case scenario of a non-luminous Jupiter-sized object occulting the white dwarf, the orbital period would have to be on the order of $3 \mathrm{yr}$ and have a semimajor axis of roughly $1.5 \mathrm{AU}$, corresponding to a transit probability of $P \sim 10^{-4}$. We conclude that the transit event we observed was far more likely caused by a short-period planet orbiting the primary star than a long-period object eclipsing the secondary.

\subsubsection{Adaptive Optics Imaging}

We also obtained high-angular-resolution imaging of the primary star to rule out any very close associated companions. We observed HIP 116454 with the Robo-AO laser adaptive optics and imaging system on the 60 inch telescope at Palomar Observatory (Baranec et al. 2014; Law et al. 2014). We obtained seven images with Robo-AO between 2014 June 15 and 2014 July 11 in three different bandpasses: Sloan $i$ band, Sloan $z$ band, and a long-pass filter with a cutoff at $600 \mathrm{~nm}$ (LP600) that more closely approximates the Kepler bandpass. Each observation consisted of a series of images read out from the detector at a maximum rate of $8.6 \mathrm{~Hz}$, for a total integration time of $90 \mathrm{~s}$. 
The frames were coadded in postprocessing using a shift-andadd technique with HIP 116454 as the tip-tilt guide star.

The quality of the Robo-AO images varied between the observations, but none of the images showed evidence for companions within three magnitudes of the primary outside of 0.2 arcsec. Some but not all of the images, however, showed an elongation that could be consistent with a bright close binary companion at a separation of 0.15 arcsec at the $<5-\sigma$ level, similar to KOI 1962 in Law et al. (2014).

To investigate this possibility further, we obtained higher resolution adaptive-optics images on 2014 August 2 using the Keck II Natural Guide Star Adaptive Optics (NGSAO) system with the NIRC2 narrow detector at Keck Observatory. We obtained unsaturated frames of HIP 116454 in $J-, H$-, and $K_{S}$-band filters to search for close companions near the diffraction limit ( $\sim 40$ mas in the $H$ band). We also acquired deeper, saturated images in the $H$ ( $70 \mathrm{~s}$ total) and $K_{S}$ bands ( $200 \mathrm{~s}$ total) with the primary positioned in the lower-right quadrant of the array and rotated so the white dwarf companion falls in the field of view. We calibrated and processed the data as described in Bowler et al. (2012). We corrected the data for optical aberrations using the distortion solution from B. Cameron (2007, private communication) and north-aligned the images using the detector orientation measured by Yelda et al. (2010). We found no evidence for the companion suggested by some of the RoboAO data. Our $7 \sigma H$-band limiting contrasts are $\{3.0,5.9,6.8$, $9.2,10.8,12.7\} \mathrm{mag}$ at separations of $\left\{0^{\prime \prime} 1,0^{\prime \prime} .3,0^{\prime \prime} .5,1^{\prime \prime} .0,2^{\prime \prime} .0\right.$, $\left.5{ }^{\prime \prime} 0\right\}$. We are able to exclude roughly equal brightness companions to an angular separation of 0.04 arcsec (projected distance of $2.2 \mathrm{AU})$.

\subsection{Reconnaissance Spectroscopy}

HIP 116454 was observed nine times for the Carney-Latham Proper Motion Survey (Latham et al. 2002) with the CfA Digital Speedometer spectrograph over the course of $9.1 \mathrm{yr}$ from 1982 until 1991. The Digital Speedometer measured radial velocities to a precision of approximately $0.3 \mathrm{~km} \mathrm{~s}^{-1}$ and detected no significant $\mathrm{RV}$ variations or trends in the velocities of HIP 116454. When corrected into an absolute RV frame, the Digital Speedometer measurements indicate an absolute $\mathrm{RV}$ of $-3.06 \pm 0.12 \mathrm{~km} \mathrm{~s}^{-1}$ and when combined with proper motion, a space velocity of $(U, V, W)=(-86.7,-0.2,4.5) \pm$ $(7.6,1.2,0.5) \mathrm{km} \mathrm{s}^{-1}$. This somewhat unusual space velocity corresponds to an elliptical orbit in the plane of the galaxy, indicating that HIP 116454 originated far from the stellar neighborhood. A detailed analysis of HIP 116454's elemental abundances could reveal patterns that are dissimilar to stars in the solar neighborhood.

We obtained three observations of HIP 116454 in June of 2014 with the Tillinghast Reflector Echelle Spectrograph (TRES) on the $1.5 \mathrm{~m}$ Tillinghast Reflector at the Fred L. Whipple Observatory. The spectra were taken with a resolving power of $R=44,000$ with a signal-to-noise ratio $(\mathrm{S} / \mathrm{N})$ of approximately 50 per resolution element. When corrected into an absolute RV frame, the TRES spectra indicate an absolute RV for HIP 116454 of $-3.12 \pm 0.1 \mathrm{~km} \mathrm{~s}^{-1}$. When combined with the absolute radial velocities from the Digital Speedometer, there is no evidence for a RV variation of greater than $100 \mathrm{~m} \mathrm{~s}^{-1}$ over the course of $30 \mathrm{yr}$. The three individual radial velocities from the TRES spectra revealed no variability at the level of $20 \mathrm{~m} \mathrm{~s}^{-1}$ over the course of 8 days. We also find no evidence for a second set of stellar lines in the cross-correlation function used to measure the radial velocities, which rules out many possible close companions
Table 2

HARPS-N Radial Velocities of HIP 116454

\begin{tabular}{|c|c|c|}
\hline BJD - 2,454,833 & $\begin{array}{c}\mathrm{RV} \\
\left(\mathrm{m} \mathrm{s}^{-1}\right)\end{array}$ & $\begin{array}{c}\sigma_{\mathrm{RV}} \\
\left(\mathrm{m} \mathrm{s}^{-1}\right)\end{array}$ \\
\hline 2012.7150 & -6.51 & 1.13 \\
\hline 2013.7062 & -3.15 & 1.37 \\
\hline 2014.7001 & -4.44 & 2.35 \\
\hline 2015.6955 & -2.62 & 1.12 \\
\hline 2016.6307 & 1.01 & 1.88 \\
\hline 2017.7029 & 4.31 & 1.24 \\
\hline 2018.6971 & 1.03 & 1.10 \\
\hline 2019.6985 & 0.33 & 1.83 \\
\hline 2020.7000 & -3.17 & 1.31 \\
\hline 2025.6645 & -0.57 & 1.22 \\
\hline 2026.6780 & -0.05 & 1.39 \\
\hline 2027.6258 & 2.33 & 1.00 \\
\hline 2028.6266 & -0.57 & 1.08 \\
\hline 2030.7261 & -0.48 & 1.19 \\
\hline 2031.7186 & -3.69 & 0.90 \\
\hline 2032.7231 & -2.82 & 1.05 \\
\hline 2033.7197 & -1.53 & 1.87 \\
\hline 2041.6353 & -1.32 & 1.28 \\
\hline 2043.6442 & -1.72 & 2.30 \\
\hline 2044.6658 & 7.28 & 1.32 \\
\hline 2045.7016 & 5.02 & 1.21 \\
\hline 2050.7205 & -5.16 & 1.41 \\
\hline 2051.7258 & -3.64 & 0.97 \\
\hline 2052.7229 & -1.40 & 1.11 \\
\hline 2053.7166 & 2.53 & 1.60 \\
\hline 2054.7304 & 4.29 & 0.92 \\
\hline 2055.6129 & 1.02 & 1.34 \\
\hline 2056.5885 & 0.20 & 1.43 \\
\hline 2057.6126 & -3.09 & 0.87 \\
\hline 2086.5397 & -1.97 & 1.07 \\
\hline 2090.5445 & 6.01 & 0.76 \\
\hline 2091.5401 & 4.58 & 0.97 \\
\hline 2098.5448 & 2.10 & 1.48 \\
\hline
\end{tabular}

or background stars. When the adaptive optics constraints are combined with a lack of RV variability of more than $100 \mathrm{~m} \mathrm{~s}^{-1}$ over $30 \mathrm{yr}$ and the lack of a second set of spectral lines in the cross-correlation function, we can effectively exclude any close stellar companions to HIP 116454.

\subsection{HARPS-N Spectroscopy}

We obtained 44 spectra of HIP 116454 on 33 different nights between July and October of 2014 with the HARPS-N spectrograph (Cosentino et al. 2012) on the $3.57 \mathrm{~m}$ Telescopio Nazionale Galileo (TNG) on La Palma Island, Spain, to measure precise radial velocities and determine the orbit and mass of the transiting planet. Each HARPS-N spectrum was taken with a resolving power of $R=115000$, and each measurement consisted of a 15 minute exposure, yielding an S/N of 50-100 per resolution element at $550 \mathrm{~nm}$, depending on weather conditions. The corresponding (formal) RV precision ranged from $0.90 \mathrm{~m} \mathrm{~s}^{-1}$ to $2.35 \mathrm{~m} \mathrm{~s}^{-1}$. Radial velocities were extracted by calculating the weighted cross-correlation function of the spectrum with a binary mask (Baranne et al. 1996; Pepe et al. 2002). In some cases, we took one 15 minute exposure per night, and in other cases, we took two 15 minute exposures back-toback. In the latter case we measured the two consecutive radial velocities individually and report the average value.

The HARPS-N RV measurements are listed in Table 2. A periodic RV variation with a period of about 9 days and a semiamplitude of about $4 \mathrm{~m} \mathrm{~s}^{-1}$ is evident in the RV time 


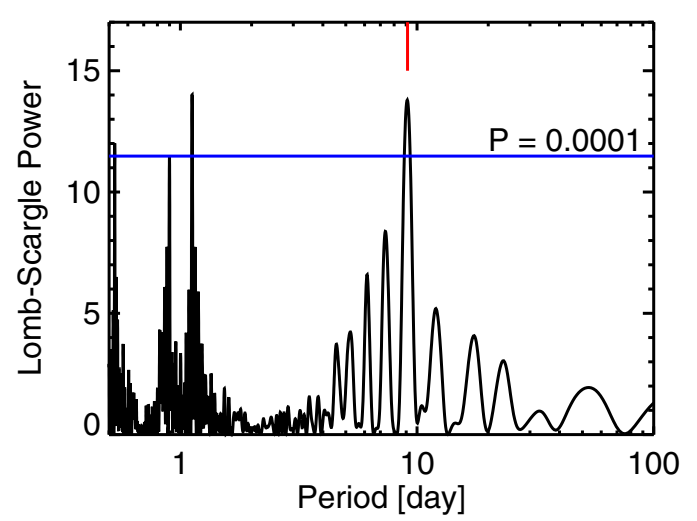

Figure 3. Left: Lomb-Scargle periodogram of the HARPS-N radial velocity data. We find a strong peak at a period of 9.1 days and see daily aliases of the 9.1 day signal with periods close to 1 day. The horizontal blue line indicates a false alarm probability of 0.0001 , and the vertical red hash mark indicates the period (9.12 days) from our combined analysis described in Section 3.2.

series. We checked that we identified the correct periodicity by calculating a Lomb-Scargle periodogram (Scargle 1982), shown in Figure 3. We found a strong peak at a period of 9.1 days and an aliased peak of similar strength with a period close to 1 day, corresponding to the daily sampling alias of the 9.1 day signal (e.g., Dawson \& Fabrycky 2010). We estimated the false alarm probability of the RV detection by scrambling the RV data and recalculating the periodogram numerous times and counting which fraction of the scrambled periodograms have periods with higher power than the unscrambled periodogram. We found that the false alarm probability of the 9.1 day periodicity is significantly less than $10^{-4}$.

In addition to the 9.1 day signal, we also found evidence for a weaker 45 day periodic RV variation. To help decide whether to include the second periodicity in our RV modeling, we fit the HARPS-N radial velocities with both a one-planet and a two-planet Keplerian model. The one-planet model was a Keplerian function parameterized by $\log (P)$, time of transit, $\log$ (RVsemiamplitude), $\sqrt{e} \sin (\omega)$, and $\sqrt{e} \cos (\omega)$, where $P$ is the planet's orbital period, $e$ is the orbital eccentricity, and $\omega$ is the argument of periastron. We also fit for a RV zero point and a stellar jitter term, for a total of seven free parameters. We fit each of these parameters with an unbounded uniform prior, except for $\sqrt{e} \sin (\omega)$ and $\sqrt{e} \cos (\omega)$, which had uniform priors over the interval between -1 and 1 . The two-planet model was the sum of two Keplerian functions, each of which was parameterized by $\log (P)$, time of transit, $\log$ (RVsemiamplitude), $\sqrt{e} \sin (\omega)$, and $\sqrt{e} \cos (\omega)$. Once again, we also fit for a RV zero point and stellar jitter term, for a total of 12 free parameters. We fit each of these parameters with an unbounded uniform prior except for $\sqrt{e} \sin (\omega)$ and $\sqrt{e} \cos (\omega)$, which had uniform priors over the interval between -1 and 1 , and for $\log P_{2}$, the period of the outer planet, which we constrained to be between the period of the inner planet and 1,000 days. We performed the fits using emcee (Foreman-Mackey et al. 2013), a Markov chain Monte Carlo (MCMC) algorithm with an affine invariant ensemble sampler. We note that upon exploring various different periods for the outer planet, our MCMC analysis found the 45 day period to be optimal. We calculated the Bayesian information criterion (BIC, Schwarz 1978) to estimate the relative likelihoods of the two models. Although the BIC does not provide a definitive or exact comparison of the fully marginalized likelihoods of the models, it allows us to roughly estimate the relative likelihoods. Upon calculating the BIC, we estimate that the two-planet model is favored over the one-planet model with confidence $P \sim 0.03$. From here on, we therefore model the RV observations as the sum of two Keplerian functions. We show our HARPS-N measurements and our best-fitting model in Figure 4.

For both periods, we find an amplitude consistent with that of a transiting super-Earth. The 9 day periodicity in the RVs is consistent with the orbital period we estimated from the duration of the K2 transit event. We "predicted" the time of transit for the 9 day period planet during the K2 observations and found that the HARPS-N measurements alone constrain the expected time of transit to better than 1 day, and we find that the $\mathrm{K} 2$ transit event is consistent with the transit ephemeris predicted by only the HARPS-N RVs at the $68.3 \%(1 \sigma)$ level. We show
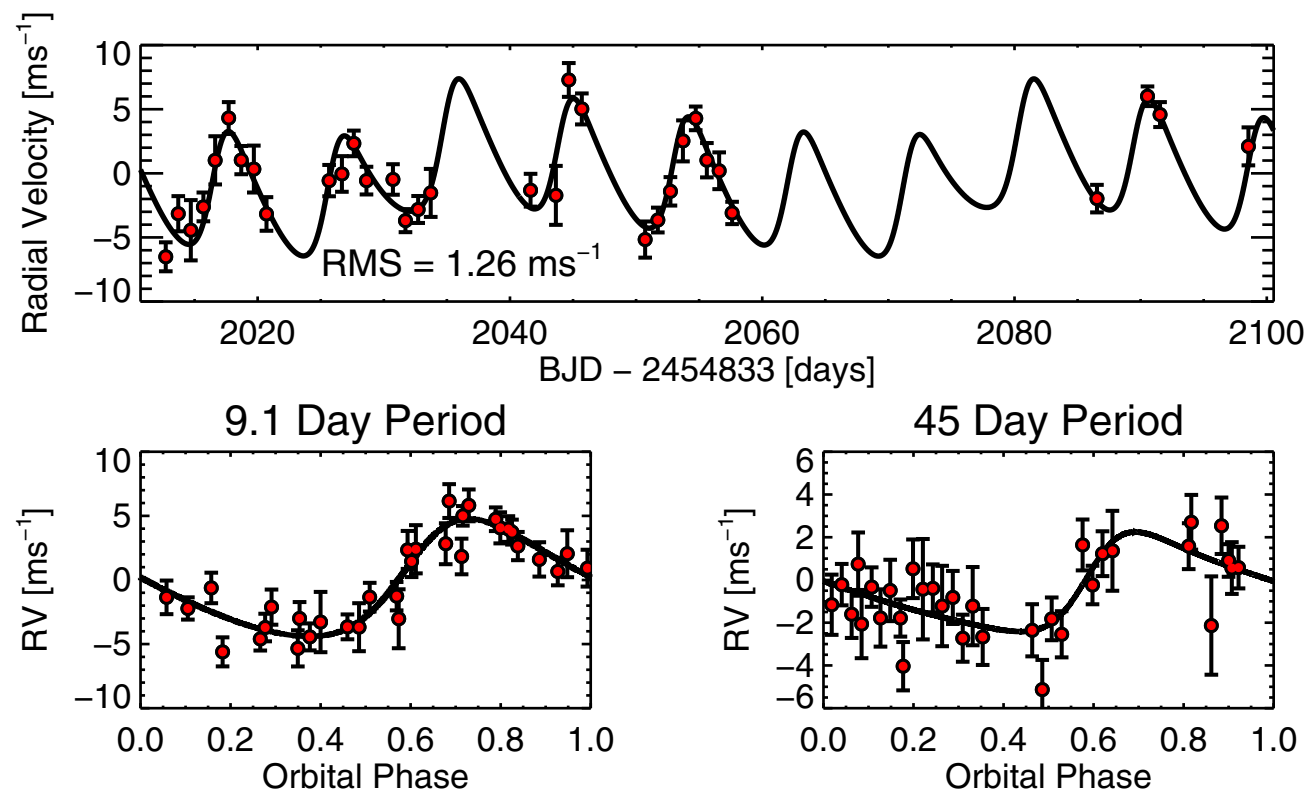

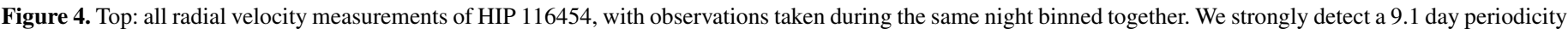

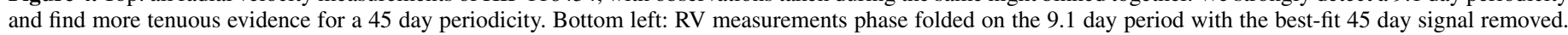
Bottom right: RV measurements phase folded on the 45 day period with the best-fit 9.1 day signal removed. 


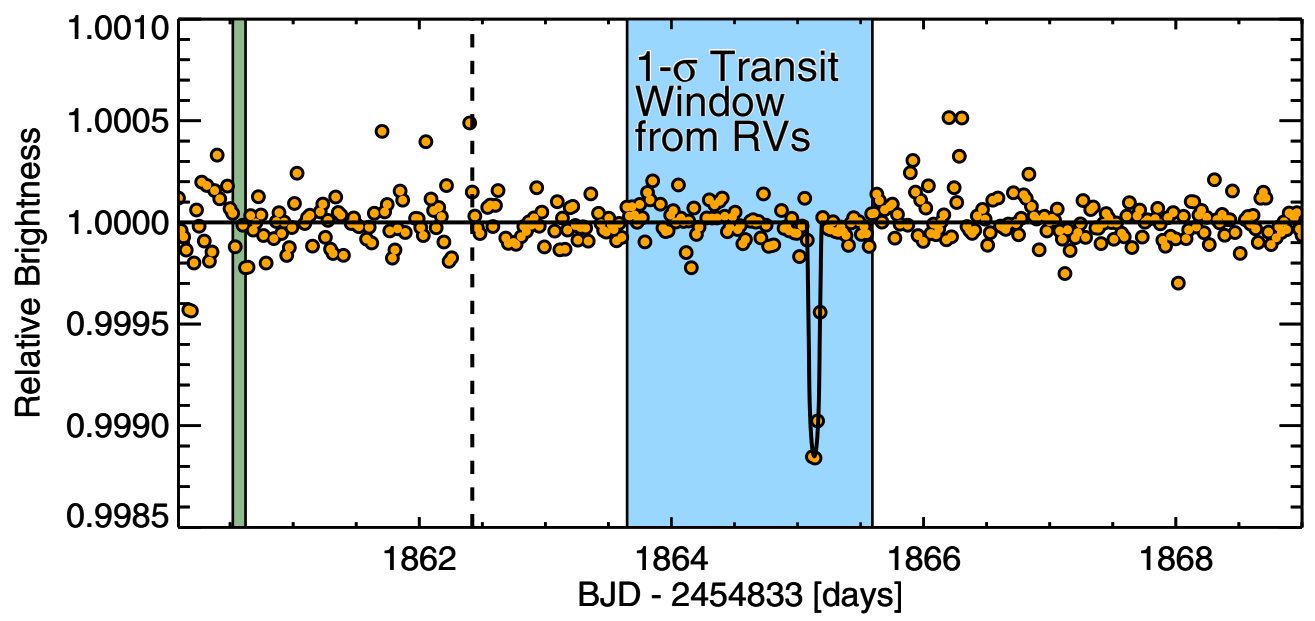

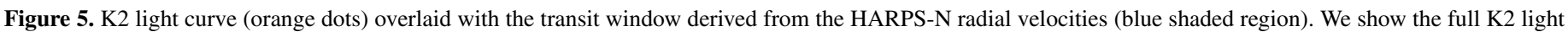

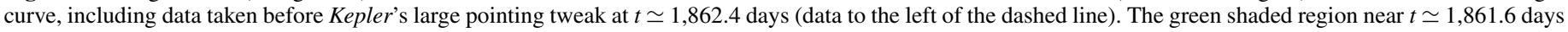

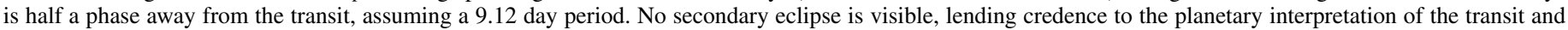
RV variations.

the K2 light curve with the transit window derived from only the HARPS-N RVs in Figure 5. Thus, the 9.1 day periodicity is consistent with being caused by a transiting planet. The more tenuous 45 day periodic variation, on the other hand, may be due to an outer planet, but it may also be caused by stellar variability.

The HARPS-N spectra include regions used to calculate activity indicators, such as the Mount Wilson $S_{\mathrm{HK}}$ index and the $R_{\mathrm{HK}}^{\prime}$ index (e.g., Wright et al. 2004). We calculated the $\mathrm{S}_{\mathrm{HK}}$ index for each HARPS-N spectrum and found a mean value of $0.275 \pm$ 0.0034 and an associated $\log _{10} R_{\mathrm{HK}}^{\prime}=-4.773 \pm 0.007$. There were no obvious correlations between the $S_{\mathrm{HK}}$ index and either the measured RV or the residuals to either a one- or two-planet Keplerian fit.

\subsection{Photometry}

\subsubsection{WASP}

HIP 116454 was observed by the SuperWASP-N instrument on La Palma, Spain, and the SuperWASP-S instrument at the South African Astronomical Observatory (Pollacco et al. 2006). The observations spanned three observing seasons, from 2008 to 2010 , and consisted of roughly 15,000 data points with a typical precision of $0.6 \%$. The WASP observations are consistent with a typical level of stellar variability at the subpercent level. The WASP data rule out deep transits but are not of high enough quality to detect the $0.1 \%$ transit depth observed by $\mathrm{K} 2$. In Section 3.1.3, we use the WASP light curve in combination with light curves from $\mathrm{K} 2$ and MOST to attempt to derive HIP 116454's rotation period.

\subsubsection{MOST}

After detecting the K2 transit, we obtained follow-up photometric observations with the MOST (Walker et al. 2003) space telescope during August and September of 2014. MOST observed HIP 116454 during three nearly continuous time spans: 13 days from 2014 August 3 to 2014 August 16, 18 days from 2014 August 21 to 2014 September 9, and 3.5 days from 2014 September 15 to 2014 September 18. During the first segment of the MOST data, observations of HIP 116454 were interleaved with observations of other stars during the satellite's orbit around Earth, but for the second and third segments MOST observed
HIP 116454 continuously. During the first and third segments, the exposure time for individual data points was $1 \mathrm{~s}$, and during the second segment, the exposure time was $2 \mathrm{~s}$.

We processed the MOST data using aperture photometry techniques as described in Rowe et al. (2006). Background scattered light (modulated by the 101 minute orbital period of MOST) and interpixel sensitivity variations were removed by subtracting polynomial fits to the correlations between the stellar flux, the estimated background flux, and the centroid coordinates of the star. At each stage, outlying data points were excluded by either sigma clipping or hard cuts. The resulting precision of the MOST light curve was approximately $0.2 \%$ per 2 s exposure.

When we search the MOST light curve at the predicted times of transits from a simultaneous analysis of the K2 and HARPS-N data, we detect a weak signal with the same depth, duration, and ephemeris as the $\mathrm{K} 2$ transit. The MOST light curve is shown in Figure 6. The MOST data refine our estimate of the transiting planet period to a precision of roughly $30 \mathrm{~s}$. We take this detection as confirmation that the 9.1 day period detected in radial velocities is in fact caused by the transiting planet. From here on, we refer to the 9.1 day period planet as HIP $116454 \mathrm{~b}$.

\section{ANALYSIS AND RESULTS}

\subsection{Stellar Properties}

\subsubsection{Spectroscopic Parameters of the Primary}

We measured the spectroscopic properties of the host star using the stellar parameter classification (SPC) method (Buchhave et al. 2012) on the spectra from TRES and HARPS-N. An analysis of spectra from both instruments showed consistent results for the stellar parameters. We adopt the results from the HARPS-N spectra because of their higher spectral resolution and S/N. The SPC analysis yields an effective temperature of $5089 \pm 50 \mathrm{~K}$, a metallicity of $[\mathrm{M} / \mathrm{H}]$ $=-0.16 \pm 0.08$, and a surface gravity of $\log g_{\star}=4.55 \pm 0.1$. We did not detect significant rotational broadening, even with the high-resolution HARPS-N spectra. The upper limit on the projected rotational velocity is roughly $v \sin (i) \lesssim 2 \mathrm{~km} \mathrm{~s}^{-1}$. 


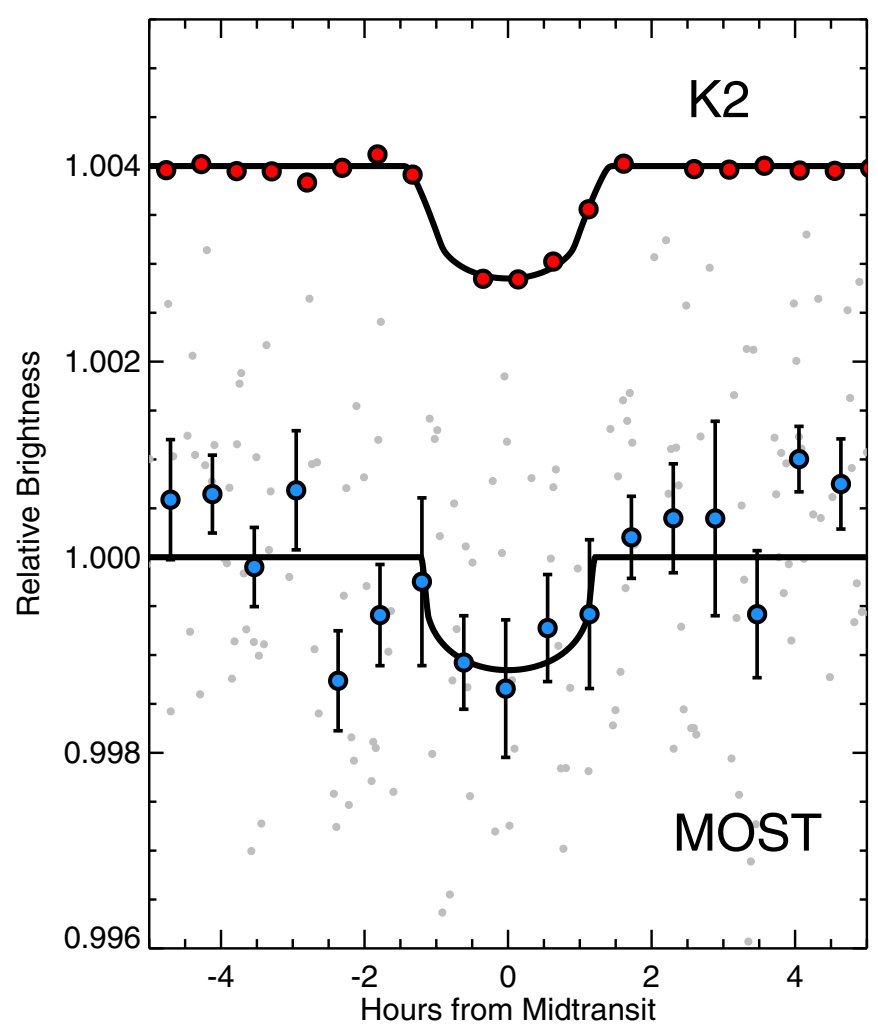

Figure 6. K2 light curve (red dots) and binned MOST light curves (blue dots). Best-fit models are overplotted in solid black lines. Individual MOST data points are shown as gray dots. The K2 light curve is vertically offset for clarity. The MOST data yield a marginal $(\simeq 3-\sigma)$ detection of the transit at the time predicted by HARPS-N radial velocities and the K2 light curve.

\subsubsection{Stellar Mass and Radius}

We used several different approaches to estimate the stellar mass and radius of HIP 116454. First, we used the SPC parameters, in particular the metallicity, surface gravity, and effective temperature, to interpolate onto the Yonsei-Yale stellar evolution model grids (Yi et al. 2001) using a Monte Carlo approach. The resulting stellar parameters were $M_{\star}=0.772 \pm 0.033 M_{\odot}$ and $R_{\star}=0.746 \pm 0.042 R_{\odot}$.

HIP 116454 was observed by Hipparcos and has a measured parallax, allowing us to interpolate model grids using a separate luminosity indicator. We used the online Padova model interpolator ${ }^{31}$, which uses the technique of da Silva et al. (2006) to interpolate a measured effective temperature, metallicity, $V$-band magnitude, and parallax onto PARSEC isochrones, yielding estimates of stellar mass, radius, surface gravity, and $B-V$ color. When the SPC parameters, Hipparcos parallax, and the $\mathrm{V}$ magnitude from the Tycho catalog (Egret et al. 1994) are provided as input, the models output $M_{\star}=0.775 \pm 0.027 M_{\odot}$ and $R_{\star}=0.716 \pm 0.024 R_{\odot}$, along with $\log g_{\star}=4.590 \pm 0.026 \mathrm{dex}$ and $B-V=0.935 \pm$ $0.018 \mathrm{mag}$.

The model-predicted surface gravity is consistent with the spectroscopically measured surface gravity and is more precise because of the additional constraint from parallax. The model output $B-V$ color is discrepant with the measured Tycho $B-V$ at the $1.5 \sigma$ level, but it is still within 0.04 mag of the measured Tycho $B-V=0.9$. This discrepancy is small enough (3\%) that

\footnotetext{
31 http://stev.oapd.inaf.it/cgi-bin/param
}

it could be due to differences in the filters used by Tycho and the filter transmission assumed by the Padova models.

We adopt the outputs from the Padova model interpolator as our stellar parameters because of their more precise constraints and ability to predict $\log g_{\star}$ and $B-V$.

\subsubsection{Stellar Rotation Period}

We attempted to measure the rotation period of HIP 116454 using photometric measurements from WASP, MOST, and $\mathrm{K} 2$ to see if stellar activity might contribute to the possible 45 day periodicity in the RV measurements. A constraint or measurement of the rotation period consistent with the possible 45 day periodicity in radial velocities could affect our interpretation of the signal. We only used photometric measurements for our analysis given the relatively short time coverage and sparseness of the spectroscopic observations.

We first started by analyzing the WASP data only because its time baseline far exceeded that of the K2 and MOST data. We binned the WASP data into nightly data points, calculated a Lomb-Scargle periodogram, and Fourier transformed the resulting power spectrum to obtain an autocorrelation function. The resulting periodograms and autocorrelation functions are shown in Figure 7. We performed this analysis on each season of WASP data individually. In the first season (2008) of WASP data, we found a moderately strong peak in both the autocorrelation function and the Lomb-Scargle periodogram at a period of about 16 days. We evaluated the significance of this peak by scrambling the binned data, recalculating the Lomb-Scargle periodograms, and counting the number of times the maximum resulting power was greater than the power in the 16 day peak. We found a false alarm probability of $2 \%$ for the peak in the first season. We did not find any convincing signals in the second (2009) or third (2010) observing seasons. A possible explanation for the inconsistency between observing seasons is that HIP 116454 experienced different levels of starspot activity, but it is also possible that the 16 day period detected in the first season is spurious. We concluded that the WASP data showed a candidate rotation period at 16 days, but the relatively high false alarm probability and lack of consistency between observing seasons precluded a confident detection.

After our analysis of the WASP data yielded suggestive yet ambiguous results, we attempted to measure the rotation period of HIP 116454 by fitting all of the photometric data with a Gaussian process noise model. Stochastic yet timecorrelated processes such as the variability produced by rotation in stellar light curves can be modeled as a Gaussian process by parameterizing the covariance matrix describing the time correlation between data points with a kernel function and inferring kernel hyperparameters from the data. We use a quasiperiodic kernel function for the specific problem of measuring a rotation period from a light curve. This in principle is better suited to inferring the rotation period of a star than a periodogram analysis because the variability produced by active surface regions on a rotating star is typically neither sinusoidal nor strictly periodic. The Gaussian process analysis also allows us to simultaneously model multiple data sets and to take advantage of data sets (like K2 and MOST) with relatively short time coverage.

We conducted our analysis using george (Foreman-Mackey et al. 2014), a Gaussian process library that employs Hierarchical Off-Diagonal Low Rank Systems (HODLR), a fast matrix inversion method (Ambikasaran et al. 2014). We used 

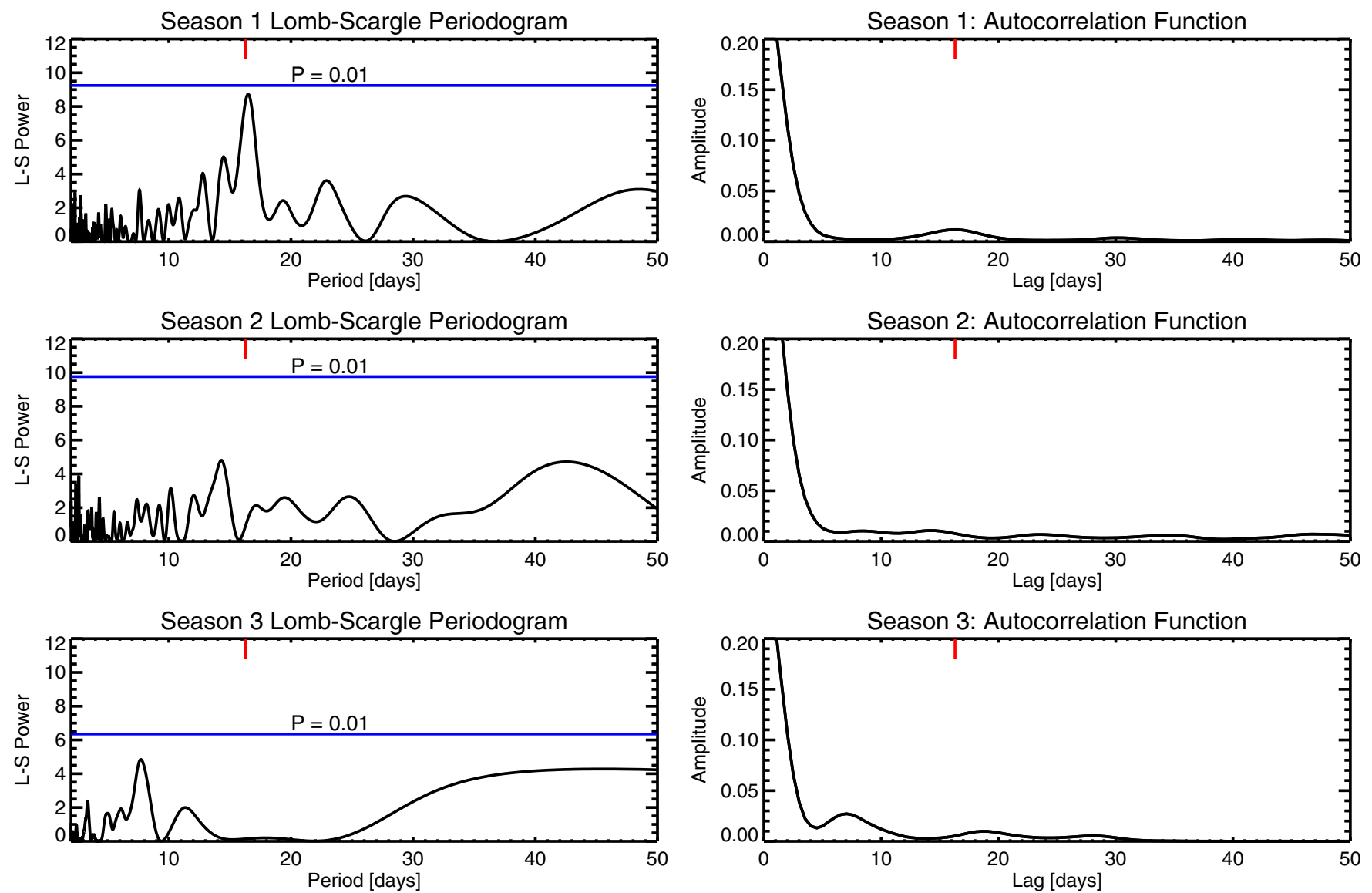

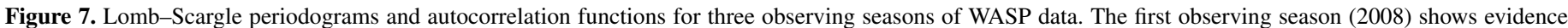

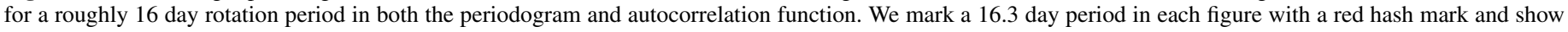
the level of a $1 \%$ false alarm probability with a horizontal blue line.

the following kernel function in our analysis:

$$
k_{i j}=A^{2} \exp \left[\frac{-\left(x_{i}-x_{j}\right)^{2}}{2 l^{2}}\right] \exp \left[\frac{-\sin ^{2}\left(\frac{\pi\left(x_{i}-x_{j}\right)}{P}\right)}{g_{q}^{2}}\right],
$$

where $A$ is the amplitude of correlation, $l$ is the timescale of exponential decay, $g_{q}$ is a scaling factor for the exponentiated sinusoidal term, and $P$ is the rotation period. An additional hyperparameter $s$ was used to account for additional white noise in the time series, where $s$ is added to the individual measurement uncertainties in quadrature.

We modeled the three continuous periods of MOST data, the three seasons of WASP data, and the K2 photometry simultaneously, with $A, l, g_{q}$, and $P$ constrained to be the same across all seven data sets and with $s$ allowed to take a different value for each. We used emcee to explore the posterior distributions of the hyperparameters. The resulting posterior distribution was not well constrained, with significant power at essentially all rotation periods greater than about 8 days and less than about 50 days. There were a few periods that seemed to be preferred to some extent in the posterior distribution: a strong peak at 12 days, and weaker peaks at 16, 20, and 32 days.

We conclude that with our data we cannot conclusively identify a rotation period for HIP 116454, which leaves us unable to rule out stellar activity as the cause of the 45 day signal in the radial velocities. While we do not find any strong evidence in the photometry that the rotation period is close to 45 days, we cannot conclusively rule out a 45 day rotation period. More photometric (or spectroscopic) observations will be important to determining HIP 116454's rotation period.

\subsection{Joint Analysis and Planet Properties}

We conducted an analysis of the K2 light curve, the HARPS-N RV observations, the MOST light curve, and the WASP light curve to determine orbital and planetary properties. We first reprocessed the $\mathrm{K} 2$ data using a different method from that described in VJ14 to minimize the possibility of any bias due to using the in-transit points in the flat field. We rederived the SFF correction by fitting the K2 light curve using an MCMC algorithm with an affine invariant ensemble sampler (adapted for IDL from the algorithm of Goodman \& Weare 2010; ForemanMackey et al. 2013). We fit the transit light curve with a Mandel \& Agol (2002) model, as implemented by Eastman et al. (2013), with quadratic limb-darkening coefficients held at the values given by Claret \& Bloemen (2011). We modeled the stellar out-of-transit variations with a cubic spline between 10 nodes spaced evenly in time, the heights of which were free parameters. Similarly, we modeled the SFF correction as a cubic spline with 15 nodes spaced evenly in "arclength," a one-dimensional metric of position on the detector as defined in VJ14. Upon finding the best-fit parameters for the SFF correction, we applied the correction to the raw $\mathrm{K} 2$ data to obtain a debiased light curve.

After rederiving the correction to the K2 light curve, we simultaneously fit a transit light curve to the K2 light curve, the HARPS-N radial velocities, and the MOST and WASP photometry using emcee. We modeled the RV variations with 
Table 3

System Parameters for HIP 116454

\begin{tabular}{|c|c|c|c|c|}
\hline Parameter & Value & & $\begin{array}{l}\text { 68.3\% Confidence } \\
\text { Interval Width }\end{array}$ & Comment \\
\hline \multicolumn{5}{|c|}{ Orbital parameters } \\
\hline Orbital period, $P$ (days) & 9.1205 & \pm & 0.0005 & A \\
\hline Radius ratio, $\left(R_{P} / R_{\star}\right)$ & 0.0311 & \pm & 0.0017 & A \\
\hline Transit depth, $\left(R_{P} / R_{\star}\right)^{2}$ & 0.000967 & \pm & 0.000109 & A \\
\hline Scaled semimajor axis, $a / R_{\star}$ & 27.22 & \pm & 1.14 & A \\
\hline Orbital inclination, $i(\mathrm{deg})$ & 88.43 & \pm & 0.40 & A \\
\hline Transit impact parameter, $b$ & 0.65 & \pm & 0.17 & A \\
\hline Eccentricity & 0.205 & \pm & 0.072 & A \\
\hline Argument of Periastron $\omega(\mathrm{deg})$ & -59.1 & \pm & 16.7 & A \\
\hline Velocity semiamplitude $K_{\star}\left(\mathrm{m} \mathrm{s}^{-1}\right)$ & 4.41 & \pm & 0.50 & $\mathrm{~A}$ \\
\hline Time of Transit $t_{t}(\mathrm{BJD})$ & 2456907.89 & \pm & 0.03 & A \\
\hline \multicolumn{5}{|c|}{ Stellar parameters } \\
\hline$M_{\star}\left(M_{\odot}\right)$ & 0.775 & \pm & 0.027 & $\mathrm{~B}, \mathrm{D}$ \\
\hline$R_{\star}\left(R_{\odot}\right)$ & 0.716 & \pm & 0.024 & $\mathrm{~B}, \mathrm{D}$ \\
\hline$\rho_{\star}\left(\rho_{\odot}\right)$ & 2.11 & \pm & 0.23 & $\mathrm{~B}, \mathrm{D}$ \\
\hline $\log g_{\star}(\mathrm{cgs})$ & 4.590 & \pm & 0.026 & B \\
\hline$[\mathrm{M} / \mathrm{H}]$ & -0.16 & \pm & 0.08 & $\mathrm{~B}$ \\
\hline Distance (pc) & 55.2 & \pm & 5.4 & $\mathrm{D}$ \\
\hline$T_{\text {eff }}(\mathrm{K})$ & 5089 & \pm & 50 & $\mathrm{~B}$ \\
\hline \multicolumn{5}{|c|}{ Planet parameters } \\
\hline$M_{P}\left(M_{\oplus}\right)$ & 11.82 & \pm & 1.33 & $\mathrm{~B}, \mathrm{C}, \mathrm{D}$ \\
\hline$R_{P}\left(R_{\oplus}\right)$ & 2.53 & \pm & 0.18 & $\mathrm{~B}, \mathrm{C}, \mathrm{D}$ \\
\hline Mean planet density, $\rho_{p}\left(\mathrm{~g} \mathrm{~cm}^{-3}\right)$ & 4.17 & \pm & 1.08 & $\mathrm{~B}, \mathrm{C}, \mathrm{D}$ \\
\hline $\log g_{p}(\mathrm{cgs})$ & 3.26 & \pm & 0.08 & $\mathrm{~B}, \mathrm{C}, \mathrm{D}$ \\
\hline Equilibrium temperature $T_{\mathrm{eff}}\left(\frac{R_{\star}}{2 a}\right)^{1 / 2}(\mathrm{~K})$ & 690 & \pm & 14 & $\mathrm{~B}, \mathrm{C}, \mathrm{D}, \mathrm{E}$ \\
\hline
\end{tabular}

Notes. (A) Determined from our analysis of the K2 light curve, the HARPS-N radial velocity measurements, the MOST light curve, and the stellar parameters. (B) Based on our spectroscopic analysis of the HARPS-N spectra. (C) Based on group A parameters. (D) Based on the Hipparcos parallax. (E) Assuming albedo of zero and perfect heat redistribution.

a two-planet Keplerian model (fitting the 9.1 day period and the 45 day period simultaneously) and modeled the transits of the 9.1 day planet with a Mandel \& Agol (2002) model. For the K2 light curve, we accounted for the 29.4-minutelong cadence exposure time by oversampling the model light curve by a factor of 13 and binning. We allowed limb-darkening coefficients (parameterized as suggested by Kipping 2013) to float. We used a white-noise model for the RV observations with a stellar jitter term added in quadrature to the HARPS$\mathrm{N}$ formal measurement uncertainties. For the light curves, we used a Gaussian process noise model, using the same kernel described in Equation (1). We used an informative prior on the stellar $\log g_{\star}$ from Section 3.1.2 in our fits, which we converted to stellar density to help break the degeneracy between the scaled semimajor axis and the impact parameter. Using this prior let us constrain the impact parameter, despite having only one K2 long-cadence data point during transit ingress and egress. In total, the model had 28 free parameters. We used emcee to sample the likelihood space with 500 "walkers," each of which we evolved through 1500 steps. We recorded the last 300 of these steps as samples of our posterior distribution, giving a total of 150,000 MCMC links. We calculated correlation lengths for all 28 parameters, which ranged from 5.6 to 19.0 , corresponding to between 8,000 and 27,000 independent samples per parameter. We assessed the convergence of the MCMC chains using the spectral density diagnostic test of Geweke (1992) and found that the means of the two sequences are consistent for 21/28 parameters $(75 \%)$ at the $1 \sigma$ level, for $27 / 28(96 \%)$ at the $2 \sigma$ level, and $28 / 28$ at the $3 \sigma$ level. These fractions are consistent with draws from a normal distribution, which is the expected behavior for the MCMC chains having converged.

In Table 3, we report the best-fitting planet and orbit parameters and their uncertainties for HIP $116454 \mathrm{~b}$ by calculating the median link for each parameter and $68 \%$ confidence intervals of all links for each parameter, respectively. We summarize the priors used in the fits and the full model outputs in Table 4, including nuisance parameters like the noise model outputs. We also make our posterior samples available for download as a FITS file.

We find that our data are best described by the presence of a planet with $R_{p}=2.53 \pm 0.18 M_{\oplus}$ and $M_{p}=11.82 \pm 1.33 M_{\oplus}$ in a 9.1205 day orbit. While we find some evidence for an outer planet in the RV measurements, we cannot conclusively claim its existence based on the data presently at our disposal.

\section{DISCUSSION}

\subsection{Composition of HIP $116454 \mathrm{~b}$}

Figure 8 shows HIP 116454 b on a mass-radius diagram with other known transiting, sub-Neptune-sized exoplanets with measured masses and radii. We overlaid the plot with model composition contours from Zeng \& Sasselov (2013). We first note that HIP $116454 \mathrm{~b}$ has a mass and radius consistent with either a low-density solid planet or a planet with a dense core and an extended gaseous envelope. The relatively low equilibrium temperature of the planet $\left(T_{\mathrm{eq}}=690 \pm 14 \mathrm{~K}\right.$, assuming zero albedo and perfect heat redistribution) makes it unlikely that any 

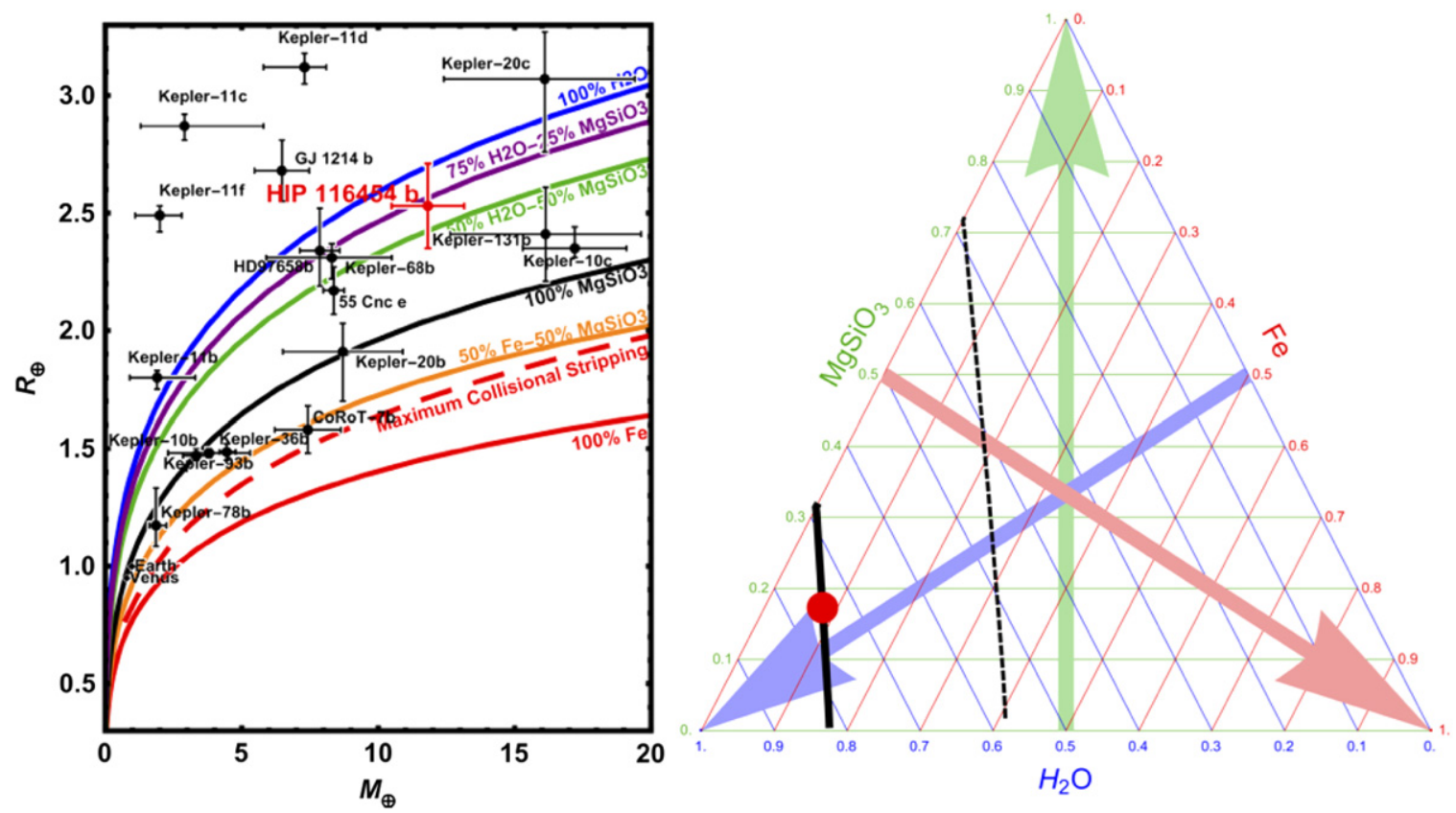

Figure 8. Left: mass-radius diagram for sub-Neptune-sized exoplanets. HIP $116454 \mathrm{~b}$ is consistent with being entirely solid, but it has a density low enough that it could also have a substantial gaseous envelope surrounding a dense core. It is similar in mass, radius, and density to HD 97658b and Kepler 68b. Right: ternary diagram showing allowed compositions for solid exoplanets. Assuming HIP $116454 \mathrm{~b}$ is solid, the thick dashed line represents allowed compositions for a planet with our best-fitting mass and radius, and the dashed line indicates the compositions allowed within $1 \sigma$ uncertainties. To $1 \sigma$, the planet must have at least $30 \%$ water or other volatiles.

Table 4

Summary of Combined Analysis

\begin{tabular}{|c|c|c|c|c|}
\hline Parameter & Prior & $50 \%$ Value & $15.8 \%$ & $84.2 \%$ \\
\hline$\sqrt{e_{1}} \cos \left(\omega_{1}\right)$ & $\mathcal{U}(-1,1)$ & 0.244 & -0.052 & +0.049 \\
\hline$\sqrt{e_{1}} \sin \left(\omega_{1}\right)$ & $\mathcal{U}(-1,1)$ & -0.395 & -0.091 & +0.109 \\
\hline$t_{t, 1},[\mathrm{BJD}]$ & $\mathcal{U}(-\infty, \infty)$ & 2456907.895 & -0.037 & +0.015 \\
\hline $\log \left(P_{1}\right.$ day $\left.^{-1}\right)$ & $\mathcal{U}(-\infty, \infty)$ & 2.210472 & -0.000176 & +0.000079 \\
\hline $\log \left(M_{1} / M_{\text {Jup }}\right)$ & $\mathcal{U}(-\infty, \infty)$ & -3.286 & -0.111 & +0.089 \\
\hline $\cos i_{1}$ & $\mathcal{U}(-1,1)$ & -0.0296 & -0.0038 & +0.0023 \\
\hline$\sqrt{e_{2}} \cos \left(\omega_{2}\right)$ & $\mathcal{U}(-1,1)$ & 0.19 & -0.26 & +0.22 \\
\hline$\sqrt{e_{2}} \sin \left(\omega_{2}\right)$ & $\mathcal{U}(-1,1)$ & -0.581 & -0.122 & +0.186 \\
\hline$t_{t, 2},[\mathrm{BJD}]$ & $\mathcal{U}(-\infty, \infty)$ & 2456930.8 & -5.4 & +5.7 \\
\hline $\log \left(P_{2}\right.$ day $\left.^{-1}\right)$ & $\mathcal{U}(-\infty, \infty)$ & 3.838 & -0.082 & +0.093 \\
\hline $\log \left(M_{2} \sin i_{2} / M_{\text {Jup }}\right)$ & $\mathcal{U}(-\infty, \infty)$ & -2.22 & -0.29 & +0.28 \\
\hline $\log R_{p, 1} / R_{\star}$ & $\mathcal{U}(-\infty, \infty)$ & -3.443 & -0.039 & +0.042 \\
\hline$q_{1}$ & $\mathcal{U}(0,1)$ & 0.37 & -0.24 & +0.33 \\
\hline$q_{2}$ & $\mathcal{U}\left(0,1-q_{1}\right)$ & 0.41 & -0.39 & +0.29 \\
\hline $\mathrm{RV}$ zero point $\left(\mathrm{m} \mathrm{s}^{-1}\right)$ & $\mathcal{U}(-\infty, \infty)$ & -0.42 & -0.34 & +0.33 \\
\hline Jitter $\left(\mathrm{m} \mathrm{s}^{-1}\right)$ & $\mathcal{U}(-\infty, \infty)$ & 0.45 & -0.28 & +0.32 \\
\hline $\log g_{\star}$ & $\mathcal{N}(4.59,0.026)$ & 4.591 & -0.020 & +0.022 \\
\hline $\log A$ & $\mathcal{U}(-20,5.5)$ & -13.05 & -0.33 & +0.35 \\
\hline $\log l$ & $\mathcal{U}(-2,8.5)$ & 1.33 & -0.93 & +1.33 \\
\hline $\log g_{q}$ & $\mathcal{U}(-8,7)$ & 2.7 & -1.6 & +1.4 \\
\hline $\log \left(P_{\text {rot }}\right.$ day $\left.^{-1}\right)$ & $\mathcal{U}(2,4)$ & 3.31 & -0.78 & +0.53 \\
\hline $\log \sigma_{\text {WASP } 1}$ & $\mathcal{U}(-6.75,-1.75)$ & -4.1 & -1.8 & +1.8 \\
\hline $\log \sigma_{\mathrm{WASP} 2}$ & $\mathcal{U}(-6.75,-1.75)$ & -4.2 & -1.8 & +1.8 \\
\hline $\log \sigma_{\text {WASP } 3}$ & $\mathcal{U}(-6.75,-1.75)$ & -4.3 & -1.7 & +1.9 \\
\hline $\log \sigma_{\mathrm{K} 2}$ & $\mathcal{U}(-11,-6)$ & -9.284 & -0.044 & +0.048 \\
\hline $\log \sigma_{\mathrm{MOST} 1}$ & $\mathcal{U}(-9,-3)$ & -6.685 & -0.059 & +0.064 \\
\hline $\log \sigma_{\mathrm{MOST} 2}$ & $\mathcal{U}(-9,-3)$ & -7.227 & -0.051 & +0.051 \\
\hline $\log \sigma_{\text {MOST3 }}$ & $\mathcal{U}(-9,-3)$ & -6.710 & -0.122 & +0.149 \\
\hline
\end{tabular}

Notes. $\mathcal{U}(A, B)$ represents a uniform distribution between $\mathrm{A}$ and $\mathrm{B}$, and $\mathcal{N}(\mu, \sigma)$ represents a normal distribution with mean $\mu$ and standard deviation $\sigma$. The limb-darkening coefficients $q_{1}$ and $q_{2}$ are defined according to the parameterization of Kipping (2013). All logarithms are base $e$.

(This table is available in its entirety in FITS format.) 
gaseous envelope the planet started with would have evaporated over its lifetime.

In terms of mass and radius, this planet is similar to Kepler$68 \mathrm{~b}\left(8.3 M_{\oplus}, 2.31 R_{\oplus}\right.$, Gilliland et al. 2013) and HD $97658 \mathrm{~b}$ (7.9 $M_{\oplus}, 2.3 R_{\oplus}$ Howard et al. 2011; Dragomir et al. 2013), but it is likely slightly larger. HIP $116454 \mathrm{~b}$ and HD $97658 \mathrm{~b}\left(T_{\mathrm{eq}} \approx\right.$ $730 \mathrm{~K})$ have similar effective temperatures, but Kepler-68 b ( $T_{\mathrm{eq}} \approx 1250 \mathrm{~K}$ under the same assumptions) is somewhat hotter. Like these planets, HIP 116454 b has a density intermediate to that of rocky planets and of ice giant planets. On the mass-radius diagram, HIP 116454 b lies close to the $75 \% \mathrm{H}_{2} \mathrm{O}-25 \% \mathrm{MgSiO}_{3}$ curve for solid planets. It could be either a low-density solid planet with a large fraction of $\mathrm{H}_{2} \mathrm{O}$ or other volatiles (which have equations of state similar to $\mathrm{H}_{2} \mathrm{O}$ ), or it could have a dense core with a thick gaseous layer.

We made inferences about the structure and composition of HIP $116454 \mathrm{~b}$ if it indeed has a dense core and thick gaseous envelope, using analytic power-law fits to the results of Lopez \& Fortney (2014). Assuming that the thick gaseous envelope is composed of hydrogen and helium in solar abundances, an equilibrium temperature calculated with perfect heat redistribution and an albedo of 0 and a stellar age of about 2 Gyr, the models predict that HIP $116454 \mathrm{~b}$ has a hydrogen and helium envelope making up about $0.5 \%$ of the planet's mass. The model suggests that HIP 116454 b has a $1.8 R_{\oplus}$ core with virtually all of the planet's mass, surrounded by a gaseous envelope with thickness $0.35 R_{\oplus}$, and a radiative upper atmosphere also with thickness $0.35 R_{\oplus}$. Using different assumptions to calculate the equilibrium temperature, like imperfect heat distribution and a nonzero albedo (for instance, the value of Sheets \& Deming 2014), and different assumptions about the envelope's composition and age does not change the calculated thickness and mass of the gaseous envelope by more than a factor of two. We note that this envelope fraction is consistent with the population of Kepler super-Earth and sub-Neptune-sized planets studied by Wolfgang \& Lopez (2014), who found the envelope fraction of these candidates to be distributed around $1 \%$ with a scatter of 0.5 dex.

We also explored the composition of HIP $116454 \mathrm{~b}$ assuming it is solid and has little in the way of a gaseous envelope, using an online tool ${ }^{32}$, based on the model grids of Zeng \& Sasselov (2013). We investigated a three-component model with layers of $\mathrm{H}_{2} \mathrm{O}, \mathrm{MgSiO}_{3}$ and $\mathrm{Fe}$. In this case, HIP $116454 \mathrm{~b}$ must have a significant fraction of either $\mathrm{H}_{2} \mathrm{O}$ or other volatiles in an ice form like methane or ammonia. The composition in this case would be more similar to the ice giants in the solar system than the rocky planets like Earth.

We find that the pressure at the core of the planet can range from $1400 \mathrm{GPa}$ for an iron-free planet to 2,800 $\mathrm{GPa}$ for a silicate-free planet. Assuming a ratio of iron to silicates similar to that of the Earth and other solar system bodies, we find that the core pressure of HIP $116454 \mathrm{~b}$ is about $2400 \mathrm{GPa}$. Under this assumption, HIP $116454 \mathrm{~b}$ would consist of $8 \% \mathrm{Fe}$, $17 \% \mathrm{MgSiO}_{3}$ and $75 \% \mathrm{H}_{2} \mathrm{O}$ by mass. Using the ratio of iron to silicates in solar system bodies is usually a relatively good assumption because this ratio is largely determined by element synthesis cosmochemistry, which does not vary greatly on the scale of 50 parsecs. However, HIP 116454's unusual space motion indicates that it might have formed elsewhere in the galaxy, so this assumption might not hold. More detailed spectral analysis, in particular measuring elemental abundances for $\mathrm{Mg}$

\footnotetext{
32 http://www.astrozeng.com
}

and $\mathrm{Si}$ compared to $\mathrm{Fe}$ in the parent star, could put additional constraints on the composition of HIP $116454 \mathrm{~b}$ assuming it is solid.

If solid, HIP $116454 \mathrm{~b}$ would be one of the " $\mathrm{H}_{2} \mathrm{O}$ rich" planets described in Zeng \& Sasselov (2014), for which it is possible to make inferences about the phase of the planet's $\mathrm{H}_{2} \mathrm{O}$ layer, given knowledge of the star's age. Various evidence points to HIP 116454 having an age of approximately 2 Gyr. Using relations from Mamajek \& Hillenbrand (2008), the $R_{\mathrm{HK}}^{\prime}$ level indicates an age of $2.7 \mathrm{Gyr}$, and the rotation indicates an age of $1.1 \mathrm{Gyr}$, if the rotation period is indeed close to 16 days. The white dwarf's cooling age, however, sets a lower limit of approximately 1.3 Gyr. Future observations, like a mass measurement of the white dwarf to estimate its progenitor's mass (and therefore age on the main sequence), could constrain the age further. If HIP 116454's age is indeed about 2 Gyr and the planet lacks a gaseous envelope, then it is likely to have water in plasma phases near its water-silicate boundary (the bottom of the $\mathrm{H}_{2} \mathrm{O}$ layer), but if it is slightly older ( $\sim 3$ Gyr or more) or has a faster cooling rate, it could have superionic phases of water.

\subsection{Suitability for Follow-up Observations}

HIP $116454 \mathrm{~b}$ is a promising transiting super-Earth for followup observations because of the brightness of its star, especially in the near infrared. We used the Exoplanet Orbit Database ${ }^{33}$ (Wright et al. 2011; Han et al. 2014) to compare HIP 116454 b to other transiting sub-Neptune-sized planets orbiting bright stars. We found that among stars hosting transiting sub-Neptunes with $R_{p}<3 R_{\oplus}$, only Kepler 37, $55 \mathrm{Cnc}$, and HD 97658 have brighter $K$-band magnitudes.

HIP 116454 is particularly well suited for additional followup photometric and RV observations, both to measure the mass of the planet to higher precision and to search for more planets in the system. HIP 116454 is chromospherically inactive and has low levels of stellar RV jitter $\left(0.45 \pm 0.29 \mathrm{~m} \mathrm{~s}^{-1}\right)$. This combined with its brightness makes it an efficient RV target. Moreover, the brightness of the host star makes HIP 116454 ideal for follow-up with the upcoming CHEOPS mission (Broeg et al. 2013).

HIP 116454 b could be important in the era of the James Webb Space Telescope to probe the transition between ice giants and rocky planets. In the solar system, there are no planets with radii between $1-3 R_{\oplus}$ while population studies with Kepler data have shown these planets to be nearly ubiquitous (Howard et al. 2012; Fressin et al. 2013; Petigura et al. 2013; Morton \& Swift 2014). Atmospheric studies with transit transmission spectroscopy can help determine whether these planets are in fact solid or have a gaseous envelope and give a better understanding of how these planets form and why they are so common in the Galaxy. Also of interest is the fact that HIP $116454 \mathrm{~b}$ is very similar to HD 97658 b in terms of its orbital characteristics (both are in $\sim 10$ day low-eccentricity orbits), mass and radius (within $10 \%$ in radius and within $25 \%$ in mass), and stellar hosts (both orbit K dwarfs). Comparative studies of these two superEarths will be valuable for understanding the diversity and possible origins of close-in super-Earths around Sun-like stars. This being said, despite HIP 116454's brightness, the relatively shallow transit depth will make it a somewhat less efficient target than super-Earths orbiting smaller stars (for instance, GJ 1214 b, Charbonneau et al. 2009).

\footnotetext{
33 http://www.exoplanets.org
} 


\subsection{Implications for K2 Science}

HIP $116454 \mathrm{~b}$ has demonstrated the potential of $\mathrm{K} 2$ to increase the number of bright transiting planets amenable to radial velocity follow-up. Despite its degraded pointing precision, it is possible to calibrate and correct $\mathrm{K} 2$ data to the point where super-Earths can be detected to high significance with only one transit. Despite the increased expense of bright stars in terms of Kepler target pixels required for the aperture, K2 data is of high enough precision to produce many transiting exoplanets around bright stars.

Many K2 fields, including the engineering test field, are located such that observatories in both hemispheres can view the stars, a significant difference between $\mathrm{K} 2$ and the original Kepler mission. Even though all of our follow-up observations for HIP $116454 \mathrm{~b}$ took place at northern observatories, the star's equatorial location enables follow-up from southern facilities like the original HARPS instrument at La Silla Observatory (Mayor et al. 2003) and the Planet Finding Spectrograph at Las Campanas Observatory (Crane et al. 2010) just as easily as with northern facilities with instruments like HARPS-N or the High Resolution Echelle Spectrograph at Keck Observatory (Vogt et al. 1994).

Many Kepler planet candidates were confirmed in part thanks to precise measurements of the Kepler image centroid as the planet transited: the expected motion of the image centroid could be calculated based on the brightness and position of other stars near the aperture, and deviations from that prediction could signal the presence of a false-positive planet candidate. Such an analysis will be substantially more difficult for K2 data because the unstable pointing leads to large movements of the image centroid. In this work, we were able to exclude the possibility of background objects creating false transit signals, taking advantage of the star's high proper motion and archival imaging. This will be more difficult for more distant stars. However, the focus of the $\mathrm{K} 2$ mission on nearby late $\mathrm{K}$ and M dwarfs, which typically have high proper motions, could make this technique of background star exclusion more widely applicable than it was for the original Kepler mission.

We thank Ball Aerospace and the Kepler/K2 team for their brilliant and tireless efforts to make the $\mathrm{K} 2$ mission a possibility and a success. Without their work, this result would not have been possible. We thank Sarah Ballard and Kevin Apps for helpful conversations. We acknowledge many helpful comments from our anonymous reviewer, as well as from Eric Feigelson, our scientific editor.

Some of the data presented in this paper were obtained from the Mikulski Archive for Space Telescopes (MAST). STScI is operated by the Association of Universities for Research in Astronomy, Inc., under NASA contract NAS5-26555. Support for MAST for non-HST data is provided by the NASA Office of Space Science via grant NNX13AC07G and by other grants and contracts. This paper includes data collected by the Kepler mission. Funding for the Kepler mission is provided by the NASA Science Mission directorate.

This research has made use of NASA's Astrophysics Data System; the SIMBAD database and VizieR catalog access tool, operated at CDS, Strasbourg, France; the Exoplanet Orbit Database and the Exoplanet Data Explorer at http://www.exoplanets.org; PyAstronomy, the repository and documentation for which can be found at https:// github.com/sczesla/PyAstronomy; and the NASA Exoplanet
Archive, which is operated by the California Institute of Technology, under contract with the National Aeronautics and Space Administration under the Exoplanet Exploration Program.

A.V. and B.T.M. are supported by the National Science Foundation Graduate Research Fellowship, grants No. DGE 1144152 and DGE 1144469, respectively. J.A.J. is supported by generous grants from the David and Lucile Packard and Alfred P. Sloan Foundations. C.B. acknowledges support from the Alfred P. Sloan Foundation. P.F. acknowledges support by Fundação para a Ciência e a Tecnologia (FCT) through Investigador FCT contracts of reference IF/01037/2013 and POPH/FSE (EC) by FEDER funding through the program "Programa Operacional de Factores de Competitividade-COMPETE." W.W.W. was supported by the Austrian Science Fund (FWF P22691-N16). The research leading to these results has received funding from the European Union Seventh Framework Programme (FP7/ 2007-2013) under grant Agreement No. 313014 (ETAEARTH). This publication was made possible through the support of a grant from the John Templeton Foundation. The opinions expressed in this publication are those of the authors and do not necessarily reflect the views of the John Templeton Foundation.

This work is based on observations made with the Italian Telescopio Nazionale Galileo (TNG) operated on the island of La Palma by the Fundacin Galileo Galilei of the INAF (Istituto Nazionale di Astrofisica) at the Spanish Observatorio del Roque de los Muchachos of the Instituto de Astrofisica de Canarias. The HARPS-N project was funded by the Prodex program of the Swiss Space Office (SSO), the Harvard University Origin of Life Initiative (HUOLI), the Scottish Universities Physics Alliance (SUPA), the University of Geneva, the Smithsonian Astrophysical Observatory (SAO), and the Italian National Astrophysical Institute (INAF), University of St. Andrews, Queens University Belfast, and University of Edinburgh.

The Robo-AO system is supported by collaborating partner institutions, the California Institute of Technology and the Inter-University Centre for Astronomy and Astrophysics, and by the National Science Foundation under grant Nos. AST-0906060, AST-0960343, and AST-1207891, by the Mount Cuba Astronomical Foundation, by a gift from Samuel Oschin.

Some of the data presented herein were obtained at the W.M. Keck Observatory, which is operated as a scientific partnership among the California Institute of Technology, the University of California, and the National Aeronautics and Space Administration. The observatory was made possible by the generous financial support of the W.M. Keck Foundation. The authors wish to recognize and acknowledge the very significant cultural role and reverence that the summit of Mauna Kea has always had within the indigenous Hawaiian community. We are most fortunate to have the opportunity to conduct observations from this mountain.

WASP-South is hosted by the SAAO and SuperWASP by the Isaac Newton Group and the Instituto de Astrofísica de Canarias; we gratefully acknowledge their ongoing support and assistance. Funding for WASP comes from consortium universities and from the UK's Science and Technology Facilities Council (STFC).

The Digitized Sky Surveys were produced at the Space Telescope Science Institute under U.S. Government grant NAG $\mathrm{W}-2166$. The images of these surveys are based on photographic data obtained using the Oschin Schmidt Telescope on Palomar Mountain and the U.K. Schmidt Telescope. The plates were processed into the present compressed digital form with the permission of these institutions. 
The National Geographic Society-Palomar Observatory Sky Atlas (POSS-I) was made by the California Institute of Technology with grants from the National Geographic Society. The Second Palomar Observatory Sky Survey (POSS-II) was made by the California Institute of Technology with funds from the National Science Foundation, the National Geographic Society, the Sloan Foundation, the Samuel Oschin Foundation, and the Eastman Kodak Corporation. The Oschin Schmidt Telescope is operated by the California Institute of Technology and Palomar Observatory.

Funding for SDSS-III has been provided by the Alfred P. Sloan Foundation, the participating institutions, the National Science Foundation, and the U.S. Department of Energy Office of Science. The SDSS-III web site is http://www.sdss3.org/.

SDSS-III is managed by the Astrophysical Research Consortium for the participating institutions of the SDSS-III Collaboration, including the University of Arizona, the Brazilian Participation Group, Brookhaven National Laboratory, Carnegie Mellon University, University of Florida, the French Participation Group, the German Participation Group, Harvard University, the Instituto de Astrofisica de Canarias, the Michigan State/ Notre Dame/JINA Participation Group, Johns Hopkins University, Lawrence Berkeley National Laboratory, Max Planck Institute for Astrophysics, Max Planck Institute for Extraterrestrial Physics, New Mexico State University, New York University, Ohio State University, Pennsylvania State University, University of Portsmouth, Princeton University, the Spanish Participation Group, University of Tokyo, University of Utah, Vanderbilt University, University of Virginia, University of Washington, and Yale University.

Facilities: Kepler, MOST, FLWO:1.5m (CfA Digital Speedometer, TRES), TNG (HARPS-N), PO:1.5m (Robo-AO), PO:1.2m, Keck:II (NIRC2)

\section{REFERENCES}

Abazajian, K. N., Adelman-McCarthy, J. K., Agüeros, M. A., et al. 2009, ApJS, 182,543

Abell, G. O. 1955, PASP, 67, 258

Akeson, R. L., Chen, X., Ciardi, D., et al. 2013, PASP, 125, 989

Ambikasaran, S., Foreman-Mackey, D., Greengard, L., Hogg, D. W., \& O’Neil, M. 2014, arXiv:1403.6015

Ballard, S., Chaplin, W. J., Charbonneau, D., et al. 2014, ApJ, 790, 12

Ballard, S., Charbonneau, D., Deming, D., et al. 2010, PASP, 122, 1341

Baranec, C., Riddle, R., Law, N. M., et al. 2014, ApJL, 790, L8

Baranne, A., Queloz, D., Mayor, M., et al. 1996, A\&AS, 119, 373

Barclay, T., Rowe, J. F., Lissauer, J. J., et al. 2013, Natur, 494, 452

Batalha, N. M., Rowe, J. F., Bryson, S. T., et al. 2013, ApJS, 204, 24

Borucki, W. J., Koch, D. G., Basri, G., et al. 2011, ApJ, 736, 19

Bowler, B. P., Liu, M. C., Shkolnik, E. L., et al. 2012, ApJ, 753, 142

Broeg, C., Fortier, A., Ehrenreich, D., et al. 2013, EPJWC, 47, 3005

Buchhave, L. A., Latham, D. W., Johansen, A., et al. 2012, Natur, 486, 375

Charbonneau, D., Berta, Z. K., Irwin, J., et al. 2009, Natur, 462, 891

Charbonneau, D., Brown, T. M., Noyes, R. W., \& Gilliland, R. L. 2002, ApJ, 568,377

Claret, A., \& Bloemen, S. 2011, yCat, 352, 99075
Cosentino, R., Lovis, C., Pepe, F., et al. 2012, Proc. SPIE, 8446, 84461V Crane, J. D., Shectman, S. A., Butler, R. P., et al. 2010, Proc. SPIE, 7735, 773553 da Silva, L., Girardi, L., Pasquini, L., et al. 2006, A\&A, 458, 609

Dawson, R. I., \& Fabrycky, D. C. 2010, ApJ, 722, 937

Dragomir, D., Matthews, J. M., Eastman, J. D., et al. 2013, ApJL, 772, L2

Dumusque, X., Bonomo, A. S., Haywood, R. D., et al. 2014, ApJ, 789, 154

Eastman, J., Gaudi, B. S., \& Agol, E. 2013, PASP, 125, 83

Egret, D., Didelon, P., McLean, B. J., Russell, J. L., \& Turon, C. 1994, yCat, 1197, 0

Foreman-Mackey, D., Hogg, D. W., Lang, D., \& Goodman, J. 2013, PASP, 125,306

Foreman-Mackey, D., Hoyer, S., Bernhard, J., \& Angus, R. 2014, george: George (v0.2.0), Zenodo, doi:10.5281/zenodo.11989

Fressin, F., Torres, G., Charbonneau, D., et al. 2013, ApJ, 766, 81

Gänsicke, B. T. 1997, PhD thesis, Universität Göttingen

Geweke, J. 1992, Bayesian Stat., 169

Gilliland, R. L., Marcy, G. W., Rowe, J. F., et al. 2013, ApJ, 766, 40

Goodman, J., \& Weare, J. 2010, Commun. Appl. Math. Comput. Sci., 5, 65

Han, E., Wang, S. X., Wright, J. T., et al. 2014, PASP, 126, 827

Howard, A. W., Johnson, J. A., Marcy, G. W., et al. 2011, ApJ, 730, 10

Howard, A. W., Marcy, G. W., Bryson, S. T., et al. 2012, ApJS, 201, 15

Howell, S. B., Sobeck, C., Haas, M., et al. 2014, PASP, 126, 398

Kipping, D. M. 2013, MNRAS, 435, 2152

Knutson, H. A., Dragomir, D., Kreidberg, L., et al. 2014, ApJ, 794, 155

Kreidberg, L., Bean, J. L., Désert, J.-M., et al. 2014, Natur, 505, 69

Latham, D. W., Stefanik, R. P., Torres, G., et al. 2002, AJ, 124, 1144

Law, N. M., Morton, T., Baranec, C., et al. 2014, ApJ, 791, 35

Lopez, E. D., \& Fortney, J. J. 2014, ApJ, 792, 1

Mamajek, E. E., \& Hillenbrand, L. A. 2008, ApJ, 687, 1264

Mandel, K., \& Agol, E. 2002, ApJL, 580, L171

Marcy, G. W., Isaacson, H., Howard, A. W., et al. 2014, ApJS, 210, 20

Mayor, M., Pepe, F., Queloz, D., et al. 2003, Msngr, 114, 20

Mestel, L. 1952, MNRAS, 112, 583

Morton, T. D., \& Swift, J. 2014, ApJ, 791, 10

Muirhead, P. S., Johnson, J. A., Apps, K., et al. 2012, ApJ, 747, 144

Pepe, F., Mayor, M., Galland, F., et al. 2002, A\&A, 388, 632

Petigura, E. A., Marcy, G. W., \& Howard, A. W. 2013, ApJ, 770, 69

Pollacco, D. L., Skillen, I., Collier Cameron, A., et al. 2006, PASP, 118,1407

Richmond, M. W., Droege, T. F., Gombert, G., et al. 2000, PASP, 112, 397

Rogers, L. A. 2014, arXiv: 1407.4457

Rowe, J. F., Matthews, J. M., Kuschnig, R., et al. 2006, MmSAI, 77, 282

Scargle, J. D. 1982, ApJ, 263, 835

Schwarz, G. 1978, Ann. Stat., 6, 461

Sheets, H. A., \& Deming, D. 2014, ApJ, 794, 133

Skrutskie, M. F., Cutri, R. M., Stiening, R., et al. 2006, AJ, 131, 1163

Teske, J. K., Cunha, K., Schuler, S. C., Griffith, C. A., \& Smith, V. V. 2013, ApJ, 778,132

Valencia, D., O’Connell, R. J., \& Sasselov, D. 2006, Icar, 181, 545

Vanderburg, A., \& Johnson, J. A. 2014, PASP, 126, 948

van Horn, H. M. 1971, in IAU Symp. 42, White Dwarfs, ed. W. J. Luyten (Cambridge: Cambridge Univ. Press), 97

van Leeuwen, F. 2007, A\&A, 474, 653

Vogt, S. S., Allen, S. L., Bigelow, B. C., et al. 1994, Proc. SPIE, 2198, 362

Walker, G., Matthews, J., Kuschnig, R., et al. 2003, PASP, 115, 1023

Wolfgang, A., \& Lopez, E. 2014, arXiv:1409.2982

Wright, J. T., Fakhouri, O., Marcy, G. W., et al. 2011, PASP, 123, 412

Wright, J. T., Marcy, G. W., Butler, R. P., \& Vogt, S. S. 2004, ApJS, 152,261

Yelda, S., Lu, J. R., Ghez, A. M., et al. 2010, ApJ, 725, 331

Yi, S., Demarque, P., Kim, Y.-C., et al. 2001, ApJS, 136, 417

Zeng, L., \& Sasselov, D. 2013, PASP, 125, 227

Zeng, L., \& Sasselov, D. 2014, ApJ, 784, 96 